\title{
Experimental study of a vortex in a magnetic field
}

\author{
Binod Sreenivasan, Thierry Alboussière \\ Department of Engineering, University of Cambridge \\ Trumpington Street, Cambridge CB2 1PZ, UK.
}

(Submitted to Journal of Fluid Mechanics)

\begin{abstract}
It is well-known that MHD flows behave differently from conventional fluid flows in two ways: the magnetic field makes the flow field anisotropic in the sense that it becomes independent of the co-ordinate parallel to the field. Secondly, the flow of liquid across the field lines induces an electric current, leading to ohmic damping. Experimental results that quantify the decay of kinetic energy and the growth of anisotropy of fully-developed MHD turbulence, when the ratio of the electromagnetic Lorentz forces to the non-linear inertial forces (termed the interaction parameter, $\left.N_{0}\right)$ assumed low to moderate $(\sim 0.1-1)$ values, are available to us. In this paper, an experimental study of the long-time decay of an initially-three-dimensional flow structure subject to a steady magnetic field, when the interaction parameter takes large as well as moderate values, is presented. This investigation is also markedly different from previous studies on quasi-2D MHD flows in thin layers of conducting fluids, where only Hartmann layer friction held the key to the dissipation of the flow.

The initial "linear" phase of decay of an MHD flow, characterized by dominant Lorentz forces and modelled extensively in the literature, has been observed for the first time in a laboratory experiment. Further, when $N_{0}$ is large compared to unity, a distinct regime of decay of a vortex follows this linear phase. This interesting trend can be explained in terms of the behaviour of the ratio of the actual magnitudes of the Lorentz to the non-linear inertial forcesthe true interaction parameter-which decreases to a constant of order unity
\end{abstract}


towards the end of the linear phase of decay, and remains invariant during a subsequent "non-linear" phase.

\section{Introduction}

Early experimental studies on MHD flows in ducts have demonstrated that a magnetic field tends to suppress turbulence in an electrically-conducting liquid (Branover

et al., 1970a; Slyusarev, 1971). It is now well-established that MHD flows differ from conventional flows in two major respects: the magnetic field elongates the flow structures in its direction so that the resulting flow field is anisotropic (see figure 1), and secondly, the flow of liquid across the magnetic field lines induces an electric current, leading to significant Joule dissipation.

Magnetic fields are commonly used to pump, stir and stabilize liquid metals. In casting operations, the motion of submerged liquid metal jets that feed the casting moulds is suppressed by the application of an intense, static magnetic field. In the absence of this "electomagnetic braking", these jets can severely disturb the free surface of the liquid, leading to the entrainment of oxides and other surface debris. In fusion tokamaks, the toroidal deuterium-tritium plasma volume is confined by a toroidal magnetic field of about 15 Tesla provided by a set of superconducting magnet coils. Between the plasma and the magnetic coils, a lithium coolant blanket is placed to absorb the energy of the emitted neutrons, to absorb the heat from the reactor core and also to breed tritium to maintain the fuel supply. The disadvantage of this scheme is that, the interaction between the intense magnetic field and the liquid metal blanket leads to suppression of turbulence, and a subsequent reduction in heat transfer rate (Lielausis, 1975). Design of effective coolant channels to overcome these adverse MHD effects is currently a very active area of research.

There is a wealth of information available in the literature on the two-dimensional nature of MHD turbulence. It was noted that the magnetic field suppressed turbulence intensities in its direction. The velocity correlation coefficients in the direction 
of the applied magnetic field were shown to approach unity, and the energy spectrum measured perpendicular to the magnetic field displayed a slope of -3 for strong magnetic fields; see Kolesnikov and Tsinober (1972), Votsish and Kolesnikov (1975). Sommeria (1988) studied two-dimensional vortices produced in a horizontal layer of mercury of thickness $a(=20 \mathrm{~mm})$ with a free upper surface, subjected to a uniform vertical magnetic field. The vortex was forced by an electric current going from an electrode in the insulating base to the electrically-conducting circular outer frame. The balance between steady electric forcing and the dissipation at the Hartmann boundary layers, and the free decay of a two-dimensional vortex due to Hartmann friction were among the cases investigated. When the current was switched off, the energy of the vortex decayed exponentially on a time scale $t_{H}$ equal to $(\rho / \sigma \nu)^{\frac{1}{2}} a / B_{0}$, solely due to dissipation in the Hartmann layer. A recent study on a quasi-2D MHD flow by Alboussière et al. (1999) features a shear layer of mercury of thickness 10 $\mathrm{mm}$ in a uniform vertical magnetic field. A radial electric current injected from a circular array of electrodes at the base of to the outer wall interacts with the magnetic field to generate a rotational motion. The swirl velocity above the bottom Hartmann layer was measured by platinum-wire potential probes embedded in the insulating base. Under a moderate magnetic field, the measured velocities were $50 \%$ of the theoretical values for laminar flow, $u_{\theta} \sim I / 4 \pi r(\sigma \rho \nu)^{1 / 2}$ (Sommeria, 1988). The authors account for the difference between the observed and predicted values in terms of the creation of a $3 \mathrm{D}$ poloidal recirculation due to Ekman pumping in the Hartmann layer. Experiments and theory agreed well in a later study performed under strong magnetic fields, indicating that the above recirculation was suppressed.

Except for the studies on energy spectra, early experimental investigations on MHD flows were aimed primarily at verifying the two-dimensional nature of MHD turbulent flow structures. Experimental investigations that quantify the long-time evolution of an initial three-dimensional flow are few and far between. A significant step in this direction was taken by Alemany et al. (1979), who investigated experimentally the decay of MHD turbulence when the initial interaction parameter took low 
$(\sim 0.1)$ as well as moderate $(\sim 1)$ values. Their configuration consisted of a grid moving through a tall vertical column of mercury subjected to an axial, homogeneous DC magnetic field. They observed that the integral length scale parallel to $\mathbf{B}$ increases with time, and the kinetic energy of the turbulence decays as $t^{-1.7}$. The latter result indicated the effect of Joule dissipation of the turbulence. The experiments were quite different from those performed earlier in ducts in a transverse field, because the measurements were practically free from wall effects. In a later study, Caperan and Alemany (1985) investigated the development of anisotropy in homogeneous MHD turbulence, and noted the transition towards a quasi-two-dimensional state.

There have also been different theoretical approaches to the problem of magnetic damping of vortical flows. The most significant ones are those by Moffatt (1967), Sommeria and Moreau (1982) and Davidson (1995). These analyses are "linear" in the sense that, the non-linear inertial terms in the equation of motion are neglected, and what results is a balance between the linear inertia, $\partial u / \partial t$ and the Lorentz forces. The assumption here is that the characteristic time during which a magnetic field eliminates velocity gradients in its direction, the Joule time $(\tau)$ is small in comparison with the turn-over time of an eddy, $t_{0}(=l / u)$ :

$$
\tau=\rho / \sigma B^{2} \ll l / u
$$

where $\rho$ and $\sigma$ are the density and electrical conductivity of the fluid, $l$ and $u$ are the typical size and velocity of the vortex, and $\mathbf{B}$ is the magnetic flux density, so that the interaction parameter, $N$, is large compared to unity:

$$
N=t_{0} / \tau=\sigma B^{2} l / \rho u \gg 1 .
$$

It is also assumed in these studies that the magnetic Reynolds number, $R_{m}$, which represents the ratio of convection to magnetic diffusion is much lesser than unity. This important dimensionless group in MHD, identified by Lehnert (1952), brings out the interplay between the flow and the magnetic field. The condition of low $R_{m}$ implies that the field induced by the motion is negligible in comparison with 
the imposed field, and the latter can be calculated as if the fluid were at rest. The condition of low $R_{m}$ is usually satisfied in laboratory experiments and industrial applications with liquid metals (Moreau, 1990). Moffatt studied the decay of the Fourier transform of the linearised, inviscid equation of motion for $\tau<t<N_{0} \tau$, where $N_{0}$ is the initial interaction parameter. The turbulent kinetic energy was shown to decay as $(t / \tau)^{-1 / 2}$. The turbulence was described as two-dimensional, in the sense that all quantities vary slowly in the direction parallel to $\mathbf{B}$ compared with their variation perpendicular to B. Sommeria and Moreau were concerned with the rate of elongation of a turbulent structure in the direction of the $\mathbf{B}$-lines. If the flow is treated as inviscid and the advection of momentum is ignored $(N \gg 1)$, the equation of motion takes the following simplified form:

$$
\frac{\partial \mathbf{u}}{\partial t}=-\frac{1}{\rho} \nabla p^{*}-\frac{1}{\tau} \nabla^{-2}\left(\frac{\partial^{2} \mathbf{u}}{\partial z^{2}}\right)
$$

where $p^{*}$ is obtained by adding the irrotational part of the Lorentz force to the fluid pressure, and $\nabla^{-2}$ is the inverse of the Laplacian operator. As the structure elongates, the gradient in the $z$-direction becomes small in comparison with that in the other two directions, so that, from equation (3), the vorticity transport equation can be written in the following approximate form:

$$
\frac{\partial \omega}{\partial t} \sim \frac{l_{\perp}^{2}}{\tau}\left(\frac{\partial^{2} \omega}{\partial z^{2}}\right)
$$

where $l_{\perp}$ is the transverse length scale. In the above equation, $l_{\perp}^{2} / \tau$ is a pseudodiffusivity of vorticity along the magnetic field lines. The length scale parallel to the field, $l_{\|}$, then evolves at a rate

$$
l_{\|}=l_{\perp}(t / \tau)^{1 / 2}
$$

From the above relation, Sommeria \& Moreau went on to suggest that, during the typical turn-over time, $l / u$, of a turbulent structure subject to a magnetic field, an anisotropic state is attained wherein

$$
l_{\|} / l_{\perp} \sim N^{1 / 2}
$$


if $l_{\perp} N^{1 / 2}$ is smaller than the spacing of the walls perpendicular to the applied field. Davidson (1995) used a result in classical electromagnetism to show that, if a flow structure evolves in a volume that is either infinite in extent or else bounded by an electrically-insulating surface, the Lorentz force cannot affect the component of angular momentum parallel to $\mathbf{B}, \mathbf{H}_{\|}$. If $l_{\perp}$ is assumed to be unaffected by the field during the initial phase of evolution, the condition of constant $\mathbf{H}_{\|}$can be coupled with the energy equation to give the laws of evolution of energy and the parallel length scale obtained by Moffatt and Sommeria \& Moreau.

During the decay of a vortex in the linear phase, the current density falls steadily. Intuitively, the Lorentz forces should weaken as time progresses, and a phase where the non-linear inertial forces are also important should ensue. An order-of-magnitude analysis performed by the authors (Sreenivasan and Alboussière, 2000) explores the decay of a flow structure in a magnetic field, after the initial linear phase has elapsed. This model is elaborated further in $\S 2$, since the results are central to our discussion in this paper.

The theories put forward for the decay of kinetic energy of an MHD flow field in the so-called linear phase await experimental validation. It is also interesting to study experimentally the transition to non-linearity of a three-dimensional flow in the linear phase, and the dependence of this transition on the initial interaction parameter. Also, if the flow is confined by boundaries normal to the external magnetic field, it would become two-dimensional at some point of time, and this time scale is again determined by the value of $N_{0}$. All the above factors have provided motivation for a new experimental study aimed at investigating the long-time evolution of a three-dimensional MHD flow field in a steady magnetic field, at large $N_{0}$. Since vortices are the buidling blocks of any turbulent flow, the study of an isolated flow structure in a magnetic field can be expected to provide a good physical insight into the behaviour of MHD turbulence. From a practical point of view, the study of a vortex offers some advantages. First, the generated vortex remains at the same 
location, enabling continuous measurement of velocity at a point during its evolution. Secondly, there are no magnetic field entry effects or field gradients across the diameter of one vortex. Finally, it is easier to realize interaction parameters greater than unity in a loaboratory with a single vortex, even with a small test section and a magnetic field of magnetic flux density are not large.

This paper is organized as follows. In $\S 2$, we review the results of the order-ofmagnitude study performed by the authors (Sreenivasan and Alboussière, 2000) on the non-linear evolution of a vortex in a magnetic field. $\S 3$ describes the experimental set-up and the measurement scheme used for studying isolated vortices in a magnetic field. In $\S 4$, we present the experimental results obtained for a range of interaction parameters and interpret them. In conclusion, we highlight the principal accomplishments of this work and discuss the implication of our results in the larger context of MHD turbulence.

\section{Non-linear decay of a vortex: the governing equations}

As mentioned in the previous section, the initial linear evolution of a liquid metal MHD flow field when $N>>1$ has been studied theoretically. However, as a flow structure elongates in the direction of the applied field, the electric current density in the core of the flow falls because currents are forced to travel along longer paths. This is also seen from the curl of Ohm's law

$$
\operatorname{curl} \mathbf{j}=\sigma \operatorname{curl}(\mathbf{u} \times \mathbf{B})=\sigma(\mathbf{B} \cdot \nabla) \mathbf{u}
$$

for a spatially-uniform magnetic field. In effect, the Lorentz forces, given by $\mathbf{j} \times \mathbf{B}$, decrease, and at one stage, they balance the non-linear inertial forces. The evolution is "non-linear" thereafter. In spectral terms, the linear phase consists of strong Joule dissipation occuring in a cone whose axis is the wave vector parallel to the magnetic field direction, $k_{\|}$. Since the energy contained in the wave vectors perpendicular to the magnetic field remains unaffected during this phase, the corresponding 
perpendicular length scale, $l_{\perp}$, can be taken to be approximately invariant. In the non-linear phase, one would expect a transfer of energy from the neighbourhood of the wave vector $k_{\perp}$ into the dissipative cone region. Intuitively, the assumption of constant $l_{\perp}$ in physical space does not hold good in this phase.

It has been proposed by the authors (Sreenivasan and Alboussière, 2000) that, during non-linear evolution, the actual ratio of the Lorentz to the inertial forces, called the true interaction parameter, $N_{t}$, is a constant of order unity. The idea essentially is that, under a steady magnetic field, the equilibrium between the Lorentz and the inertial forces is preserved. If this argument is used in conjunction with the principle of conservation of the parallel component of the angular momentum (Davidson, 1995) and the kinetic energy equation, the non-linear vortex evolution can be fully described. The global angular momentum is defined as

$$
\mathbf{H}=\rho \int_{V} \mathbf{x} \times \mathbf{u} d V .
$$

The density, $\rho$, being constant and uniform, the conservation of the component of angular momentum parallel to the magnetic field, $\mathbf{H}_{\|}$, can be written as follows:

$$
E^{1 / 2} l_{\perp}^{2} l_{\|}^{1 / 2}=\text { const. }
$$

where $E$ is the global kinetic energy of the vortex $\left(\sim \int_{V} u^{2} d V\right)$. The kinetic energy equation and the condition of $N_{t}$ being of order unity are respectively,

$$
\begin{aligned}
\frac{\mathrm{d} E}{\mathrm{~d} t} & \sim-\frac{E}{\tau}\left(\frac{l_{\perp}}{l_{\|}}\right)^{2} \\
N_{t} & =N\left(\frac{l_{\perp}}{l_{\|}}\right)^{2} \sim 1
\end{aligned}
$$

The right hand side of the energy equation (10) represents Joule dissipation, and is obtained by estimating the current density from Ohm's law. An order-of-magnitude study of the energy decay of the vortex shows that viscous damping is negligible in comparison with ohmic damping, and could been dropped in the analysis. The true interaction parameter is expressed in the form given in relation (11) by estimating the actual magnitudes of the Lorentz and the inertial forces. Solution of the equations 
(9), (10) and (11) gives the following laws for decay of energy and growth of length scales, at large times $(t>>\tau)$ :

$$
E \sim(t / \tau)^{-1}, l_{\|} / l_{0} \sim(t / \tau)^{3 / 5}, l_{\perp} / l_{0} \sim(t / \tau)^{1 / 10}
$$

From the above estimates, one can obtain the following non-linear decay law for the ensemble average of the square of the vortex velocity, $\overline{u^{2}}$, which is typically measured in a laboratory experiment:

$$
\overline{u^{2}} \sim E / l_{\perp}^{2} l_{\|} \sim(t / \tau)^{-9 / 5}
$$

From the expression for the true interaction parameter (11), it can be readily shown that

$$
N_{t} \sim N_{0}(t / \tau)^{-1 / 2}
$$

Hence, $N_{t}$ actually decreases with time during the linear phase, and when $t \sim N_{0}^{2} \tau$, the evolution enters the non-linear regime. This model provides the laws of evolution of a vortex in both the linear and non-linear regimes.

Moffatt (1967) had initially proposed that the characterisitc duration of the linear decay phase of MHD turbulence is $N_{0} \tau$, or simply, the turn-over time. It was Alemany et al. who put forward a more precise estimate of the duration of this phase. By equating the time scales for ohmic dissipation of the eddies and the time taken for non-linear transfer of energy into the dissipative cone region (in Fourier space), they had obtained the estimate $t \sim N_{0}^{4 / 3} \tau$. However, for an isolated vortex, we have found that the linear phase lasts until $t \sim N_{0}^{2} \tau$. A direct orderof-magnitude calculation similar to that in relation (14) can also be performed for MHD turbulence, by noting that the kinetic energy per unit mass, given by the mean square value of the velocity, $\overline{u^{2}}$, follows the $(t / \tau)^{-1 / 2}$ law. The estimate of Alemany et al. follows.

In the next two sections, we present the details of an experiment to observe the decay of a single vortex. 


\section{Experimental set-up}

\subsection{Test facility}

A new experimental test facility was set up to study the long-time evolution of vortices in a liquid metal subject to a uniform magnetic field. The set-up essentially consists of (i) a water-cooled electromagnet powered by a $32 \mathrm{~kW}$ DC power supply unit, to supply the desired magnetic field up to a maximum of 1 Tesla, (ii) a current pulser to generate vortices in the liquid metal subject to this magnetic field, (iii) potential probes for velocity measurement and (iv) a high-speed automated dataacquisition system to capture the transient decay phase. The flux density is uniform within $4 \%$ over a length of $200 \mathrm{~mm}$, a breadth of $100 \mathrm{~mm}$ and a depth of $100 \mathrm{~mm}$ in the air gap of the magnet. The test section, of inside dimension $200 \times 80 \times 80$ $\mathrm{mm}$, and made of insulating Plexiglas (figure 2), is located centrally between the pole pieces of the magnet. The box contains mercury of purity $99.99 \%$ by weight (density, $\rho=13.55 \times 10^{3} \mathrm{~kg} \mathrm{~m}^{-3}$, electrical conductivity, $\sigma=1.04 \times 10^{6} \Omega^{-1} \mathrm{~m}^{-1}$ ). Though the unit is sealed at the top and bottom with O-rings, pure argon is bled over the free surface of the mercury at periodic intervals, to prevent oxidation and subsequent loss of electrical contact.

The study of the decay of a vortex in a box with electrically-insulating boundaries is probably the closest real life simulation of a vortex evolving freely in an infinite domain. The presence of conducting walls normal to the magnetic field lines would mean that there is no gradient of potential at the wall to balance $\mathbf{u} \times \mathbf{B}$, and the significant current density causes rapid ohmic damping of the flow; see Platnieks and Freiberg (1971). More formally, if there is a net flux of electric current across the boundaries of the domain, the angular momentum of a vortex is not conserved, and the theoretical decay laws would no longer be valid (Davidson, 1995). 


\subsection{Vortex generation mechanism}

Several stainless steel electrodes of diameter $1 \mathrm{~mm}$ are located flush with the polished inside surface of one of the walls of the test section to generate isolated vortices. Vortices are created in the quiescent liquid metal by pulsing an electric current from a central positive electrode to four surrounding negative electrodes. Figure 3 shows the front view and plan of one set of electrodes used for the purpose. The power source of the current pulser consists of 12, 2.5 Ampere-hour, rechargeable lead-acid cells. The unit is capable of supplying a maximum of $100 \mathrm{~A}$, at $24 \mathrm{~V}$, for a maximum duration of 100 millisecond. The pulse width is controlled by a monostable, and the current passing through the mercury is monitored across an external resistor that produces a potential drop of $20 \mathrm{mV} / \mathrm{A}$ across it. The injected current interacts with the magnetic field, $B$, to give rise to a localised swirl motion within the region of injection. The acceleration of the liquid, due to the Lorentz forces acting on it during injection of electric current is given by

$$
\frac{\partial u}{\partial t}=(\mathbf{j} \times \mathbf{B}) / \rho
$$

and hence, the characteristic swirl velocity generated in the liquid can be estimated by integrating the above equation:

$$
u_{\theta} \sim \frac{t_{p} I B}{\rho l^{2}}
$$

where $t_{p}$ is the current pulse-width in second, $I$ is the injected current in Ampere and $l$ is the electrode spacing in metre. The duration of the current pulse, $t_{p}$, is chosen so as to be generally smaller than the Joule time. This ensures that the vortex generation phase does not influence the decay phase of the vortex i.e. the generated vortex is not elongated in the direction of the field prior to measurement of its decay. Thus, a evolution of a freely-decaying vortex can be studied. For a magnetic field of $0.5 \mathrm{~T}$ acting on mercury, the Joule time is about 50 millisecond, and a current pulse duration of 10 millisecond would be appropriate. However, too

small a pulse width cannot be chosen because the ratio of the characteristic diffusion 
time of the magnetic field to the period of the pulse, called the shielding parameter (Moreau, 1990), would be large compared to unity. In such a case, the penetration of the injected electric current (and the corresponding magnetic field perturbations) on a time scale of $t_{p}$ would be confined to a thin layer adjacent to the wall. As a consequence, the generated vortex would be flat, and not spherical.

For a characteristic length scale of $0.01 \mathrm{~m}$, and a pulse width of 10 millisecond, the shielding parameter is

$$
R_{\omega}=\mu \sigma \omega_{p} l^{2}=0.013(\ll 1)
$$

where $\omega_{p}=1 / t_{p}$. The depth of penetration into the liquid metal during this pulse duration is

$$
L_{p} \sim\left(t_{p} / \mu \sigma\right)^{1 / 2}=0.09 \mathrm{~m}
$$

Thus, the problem of limited penetration, the so-called the skin effect does not occur in our experiments.

The technique of using electric currents to generate vortices in an electricallyconducting fluid is not new; see Sommeria (1988); Tabeling et al. (1991). The experiments reported in this paper are different from those performed earlier by Sommeria (1988) and Alboussière et al. (1999) — the initial size of a vortex being typically $10 \mathrm{~mm}$, the spacing between the walls normal to the magnetic field is 80 $\mathrm{mm}$. We are primarily concerned with the transition from a fully three-dimensional state to an anisotropic, and then a final two-dimensional state, wherein the parallel length scale of the structure is of the order of the width of the cavity. At reasonably large Hartmann numbers, one expects the Joule dissipation due to induced currents in the core of the flow to play a major role in damping of the vortex, and Hartmann friction to be significant only when two-dimensionality in the sense described above has been reached (see Appendix A.2 for a detailed development). 


\subsection{Instrumentation and data-logging}

If the measured potential difference is of the order of microvolts, thermoelectric voltages arising from the use of dissimilar metals can corrupt the signal. Platinum is ideally suited for potential measurements in mercury because the absolute thermoelectric powers (in $\mu \mathrm{V} /{ }^{\circ} \mathrm{C}$ ) of both metals are approximately equal at room temperature. The tips of the probes were cleaned with methanol and then sputtered with a layer of gold a few nanometres thick. As gold comes into contact with mercury, it dissolves, exposing the platinum surface underneath. Measurement probes are located in the region of vortex formation as shown in figure 3 , and spaced 2.5 $\mathrm{mm}$ apart. The potential difference between probes $a$ and $b$, and that between $b$ and $c$, should give estimates for two components of the velocity. These probes also provide information on the velocity correlation between the opposite walls. If the velocity correlation coefficient measured from probes aligned with the magnetic field lines and located on opposite walls rises steeply to unity, we can say that the flow structure has felt the presence of the boundaries of the domain, and has become nearly two-dimensional.

In addition to the above static probes, a set of axially-movable internal potential probes were provided with the intention of measuring the velocity correlation between different points in the core of the flow, aligned with the magnetic field lines (see figure 2). These data could possibly provide information on the growing length scale of the vortex, and thus, the degree of anisotropy.

The potential difference measured by two probes is fed as a differential input to a low-noise instrumentation amplifier of gain 1000, and the output wires are taken as twisted, shielded pairs to the data acquisition system. Data is acquired by a 16-bit resolution, PCI-series card capable of sampling at $10^{5}$ samples per second over 32 differential-input channels. One of the digital I/O lines of the card was configured to drive the current pulser unit. The entire process of current injection and acquisition was programmed using the software LabVIEW. One realization comprises the 
following events: (a) measurement of offsets in every channel (so that variations with time, if any, can be taken care of), (b) injection of a single current pulse and simultaneous acquisition of the voltage signals at the rate of at least a 1000 samples per second, for a period of 2 seconds and $(c)$ a time delay of 60 seconds to enable the liquid to become quiescent before the next realization.

\section{Experimental results}

The focus of the experimental study is on understanding the influence of the initial interaction parameter, or the relative magnitudes of the Lorentz and the inertial forces, on the evolution pattern. If both the linear and non-linear phases of decay are to be captured in one experiment, one should aim to achieve an interaction parameter sufficiently greater than unity. It should also be possible to suitably reduce the value of $N_{0}$ to identify the case when the two forces are of the same order of magnitude from the beginning of an experiment. From the estimate of the initial swirl velocity of the vortex given by equation (16), the following estimate of the initial interaction parameter can be obtained:

$$
N_{0}=\frac{\sigma B^{2} l}{\rho u_{\theta}} \sim \frac{\sigma B l^{3}}{t_{p} I},
$$

where $l, I$ and $t_{p}$ have notations as in relation (16). Based on the value of $N_{0}$ desired, suitable values of $B$ and $t_{p}$ can be chosen (the latter has to be shorter than the Joule time). This gives the intensity of current $I$ to be used. For example, the case $N_{0}=3$ can be realized with a flux density of $0.6 \mathrm{~T}$, a pulse width of 10 millisecond and a current of $20 \mathrm{~A}$. The corresponding velocity calculated from the estimate (16) is $0.09 \mathrm{~ms}^{-1}$. The data obtained for these initial conditions are studied first.

\subsection{Acquired data}

Figure 4 shows the data in millivolt recorded by the probe pair $a-b$ in one realization, after digital low-pass filtering of the raw data at $30 \mathrm{~Hz}$. This cut-off ensures removal 
of $50 \mathrm{~Hz}$ and high-frequency noise that otherwise corrupts our low-frequency (1-20 $\mathrm{Hz}$ ) signal. The time axis in the figure is normalized with respect to the Joule time. In figures 5 and 6 , the sum of the squares of the two potentials measured at the wall of injection (that correspond to two components of the velocity) is ensembleaveraged. The square of the potential averaged over 5, 10 and 20 realizations of the flow, is shown for $N_{0}=3$. The ranges $2<t / \tau<10$ and $10<t / \tau<60$ are shown separately in two figures for clarity. The $y$-axis is normalized with respect to the ensemble average of the value at $t=\tau, \overline{(\Delta \phi)_{0}^{2}}$. This "reference" value, used in all subsequent graphs, corresponds to the potential difference measured immediately after the current-injection phase, and does not represent $\overline{u_{0}^{2}}$ accurately. In fact, the initial velocity of the vortex cannot be deduced from potential measurements owing to the significant electric currents present in the core of the flow. In the first phase, the curves are almost similar, characterised by oscillations. These oscillations can possibly be the signature of an initial non-axisymmetry of the vortex, because there are only four outer electrodes to receive the injected current. With a circular ring of electrodes surrounding the central one, better axisymmetry is possible, and the fluctuations observed in the curve may disappear. The evolution in the initial phase is deterministic, since the above behaviour of the curve is reproduced irrespective of the number of realizations taken. The study of any one realization of the flow would suffice to understand the typical evolution of the vortex in this regime. In the second range, the behaviour is chaotic, and changes with the number of realizations considered. The curve corresponding to 5 realizations has more fluctuations in it, and shows a steeper slope. Though the curves corresponding to 10 and 20 realizations are nearly identical, the one obtained with 20 realizations is smoother. The amplitude of the random fluctuations superposed on the mean signal is reduced by averaging. In the results presented in $\S 4.3$, average over 20 realizations is considered. Before proceeding with a quantitative study of the averaged data in the two regimes of evolution and their comparisons with theory, a note on the estimation of velocities from wall-potential measurements is appropriate. 


\section{$4.2(\nabla \phi)$ as a measure of $u$}

Researchers in the past (Branover et al., 1970a,b; Kit, 1970) have used potential probes for measurement of turbulence intensities in mercury contained in a rectangular duct, subject to a transverse magnetic field. Knowing that the magnetic field suppresses velocity gradients in its direction, they have assumed that the current density in the core of the flow is small, so that $\nabla \phi$ balances $\mathbf{u} \times \mathbf{B}$. Platnieks (1971), in a comparative study of hot-wire anemometric and potential measurements of turbulence intensities in liquid metals, reported good agreement between results obtained by the two methods for $H a>50$, where $H a$ was based on the width of the channel under consideration.

The probes located on the wall of the test section are expected to give information on two components $(x$ and $y$ ) of the velocity in a plane transverse to the magnetic field, B. Since $u_{z}$ is in the direction of B, Ohm's law precludes its measurement. If any probes aligned with the magnetic field were located in the core of the flow, they would measure only the current $j_{z}$. For an axisymmetric, swirling vortex, there is no potential variation in the $\theta$-direction. Thus, the measurement of $u_{r}$ from circumferential measurements of $(\nabla \phi)$ is also ruled out. The only component of velocity that can be measured is $u_{\theta}$. The rate of decay of $E_{\theta}$ being nearly identical to that of the total energy, $E$ even for a fully non-linear evolution (Sreenivasan, 2000), the measurement of $u_{\theta}$ alone should be adequate to understand the evolution of the total mean square velocity, $\overline{u^{2}}$. Probes $a-b$ lie exactly along a radius, hence, they are ideal for the measurement of $u_{\theta}$ (see figure 3). Ohm's law, in the form given by equation (7), gives an estimate of the "parallel" component of the electric current as

$$
\mathbf{j}_{\|} \sim \sigma B \mathbf{u}\left(\frac{l_{\perp}}{l_{\|}}\right)
$$

and by the condition of current conservation, the radial component of the current is estimated by

$$
j_{r} \sim-\sigma B u_{\theta}\left(\frac{l_{\perp}}{l_{\|}}\right)^{2} .
$$


Now, the radial component of Ohm's law can be written as

$$
\begin{aligned}
-\sigma B u_{\theta}\left(\frac{l_{\perp}}{l_{\|}}\right)^{2} & \sim \sigma\left[-(\nabla \phi)_{r}+u_{\theta} B\right], \text { or } \\
(\nabla \phi)_{r} & \sim u_{\theta} B\left[1+\left(\frac{l_{\perp}}{l_{\|}}\right)^{2}\right] .
\end{aligned}
$$

When $t=5 \tau,\left(l_{\|} / l_{\perp}\right) \approx 2$, so the velocity is estimated with an error of $20 \%$. When the flow structure has propagated over half the width of the test cell $\left(l_{\|} \approx 40 \mathrm{~mm}\right)$, the error is of order $1 / 16$. The potential measurement thus gives a fairly good estimate of the velocity after the first few Joule times. The results shown in $\S 4.3$ are based on the potentials measured at the two walls of the test section, but in the light of the above discussion, we take the liberty of expressing the potentials in terms of the respective velocities, so as to give a better physical feel for the reader.

Another approximation commonly associated with wall-potential measurements is that the jump in potential across the Hartmann layer is negligible, so that the distribution of electric potential in the core region and the Hartmann walls is nearly identical (see, for example, Burr et al. (2000)). Though this assumption may well be true, an order-of-magnitude analysis should verify this. The current conservation condition for the Hartmann layer can be integrated over the thickness of the layer, $\delta(\sim 1 / H a)$ to give

$$
j_{\|}=-\frac{L}{H a} \frac{\partial j_{\perp}}{\partial l_{\perp}}
$$

where $L$ is the spacing between walls. Now, since the parallel component of the current is directly obtained from the parallel gradient of potential i.e.

$$
j_{\|}=-\sigma\left(\frac{\partial \phi}{\partial z}\right)_{H a}
$$

the potential difference across the Hartmann layer is essentially

$$
(\Delta \phi)_{H a} \sim \frac{1}{\sigma} \frac{L^{2}}{H a^{2}} \frac{j_{\perp}}{l_{\perp}}
$$

$j_{\perp}$ cannot be greater than $\sigma B u_{c}$, where $u_{c}$ is the velocity in the core of the flow. The latter can be taken as a conservative estimate of $j_{\perp}$. Hence, the ratio of the potential 
drop across the Hartmann layer to that measured at the wall between probes $a$ and $b$ in figure 3 can be written as follows:

$$
\frac{(\Delta \phi)_{H a}}{(\Delta \phi)_{a b}} \sim\left(\frac{L}{H a}\right)^{2} \frac{1}{\delta_{a b} l_{\perp}}
$$

where $\delta_{a b}$ is the potential probe spacing at the wall. In the present experiment, $L=80 \mathrm{~mm}, l_{\perp} \approx 10 \mathrm{~mm}$ and $\delta_{a b}=2.5 \mathrm{~mm}$. Even for a magnetic field of $0.1 \mathrm{~T}$, the above ratio is of order $10^{-2}$. For the range of magnetic fields considered in this study $(0.4-0.9 \mathrm{~T})$, the gradient in potential across the Hartmann layer will certainly be small.

\subsection{Effect of $N_{0}$ on vortex evolution}

The decay phase of a vortex at $N_{0}=3$, when studied in isolation (see figure 7 ), reveals the interesting trend of two different rates of decrease of $\overline{u^{2}}-$ one, a decay rate of $(t / \tau)^{-1}$ in the range $3 \tau<t<10 \tau$, and two, a rate of $(t / \tau)^{-9 / 5}$ until $t \approx 30 \tau$. The former phase corresponds to the "linear" evolution described in $\S 1$, because a decay of $(t / \tau)^{-1 / 2}$ for the total energy, and a growth rate of $(t / \tau)^{1 / 2}$ for the parallel length scale implies an evolution law of $\sim(t / \tau)^{-1}$ for $\overline{u^{2}}$. The latter phase must be non-linear, because the rate of decay is equal to the rate of decay of $\overline{u^{2}}$ predicted in $\S 2$, equation (13). The experimental result departs from the theoretical prediction at small Joule times, since the method of estimation of the velocity by potential measurement fails due to the presence of significant induced electric currents in the core of the flow. Also, the linear decay law $E \sim(t / \tau)^{-1 / 2}$ is expected to hold good only at times greater than $\tau$. The good agreement observed after $t \approx 3 \tau$ is a clear indication of the fact that the magnitude of the current density in the core has fallen to a value low enough to make $\nabla \phi$ of the same order as $\mathbf{u} \times \mathbf{B}$.

The predicted law for the non-linear phase is not valid for $t>25 \tau$, because further evolution is limited by the presence of the walls normal to the field. The attainment of a 'two-dimensional' state is evident from the rise of the velocity correlation coefficient curve (shown on the right ordinate in figure 7, in linear scale), which occurs 
nearly at the same time as the departure of the experimental curve of $\overline{u^{2}}$ from the theoretical line. Figure 8 shows the results for $N_{0}=3$, achieved by generating a higher initial velocity field, and imposing a suitably-higher magnetic field. The trend is very similar to that observed in figure 7 . This result is significant because it tells us that the evolution is dependent only on the value of $N_{0}$.

As $N_{0}$ increases, one can observe that both the linear and non-linear phases last longer (in terms of Joule times). The fluctuations observed in the signal at higher field strengths can be attributed to the noise generated by the power transformer being superposed on the signal. If the Lorentz forces are significantly larger than the non-linear inertial forces, the vortex should evolve for more number of Joule times before the fall of electric current in the core brings the two forces to a state of equilibrium. Further, the rate of growth of the parallel length scale of the vortex is lower during the linear phase compared to that in the non-linear phase, so that there is enough scope for elongation of the vortex in the non-linear phase until $l_{\|}$ is equal to the transverse dimension of the cavity $\left(80 \mathrm{~mm}\right.$ ). For $N_{0}=7.7$ (figure 11), the longest possible linear phase is obtained in the experiment (that lasts until $t \approx 50 \tau)$. A two-dimensional state is attained when $t \approx 70 \tau$. On the other hand, for $N_{0}=1$ (see figure 13), the evolution begins with the non-linear phase, and lasts only for about 12 Joule times before the flow structure meets the opposite wall. The absence of the linear phase leads to the conclusion that the inertial and Lorentz forces are of the same order of magnitude initially. It may be noted that even for $N_{0}=1.4$, there exists a linear phase preceding the non-linear evolution (figure 12).

Table 1 summarizes the initial conditions, the dimensionless groups and the approximate values of the time scales measured in the experiments. Here, $u_{0}$ is the estimated initial velocity, $R e$ is the initial Reynolds number based on the vortex diameter, and $R e_{H a}$ is the Reynolds number based on the thickness of the Hartmann layer. The subscript $l$ refers to the end of the linear phase, and $n l$, the termination of the non-linear phase enforced by the walls of the test section. 


\begin{tabular}{|c|c|c|c|c|c|c|c|c|c|c|}
\hline$B, \mathrm{~T}$ & $\tau, \mathrm{s}$ & $u_{0}, \mathrm{~ms}^{-1}$ & $R e$ & $N_{0}$ & $(t / \tau)_{l}$ & $t_{l}, \mathrm{~s}$ & $(t / \tau)_{n l}$ & $t_{n l}, \mathrm{~s}$ & $H a$ & $R e_{H a}$ \\
\hline \hline 0.92 & 0.015 & 0.085 & 7460 & 7.7 & 50 & 0.77 & 70 & 1.08 & 1900 & 30 \\
0.85 & 0.018 & 0.094 & 8250 & 5.9 & 25 & 0.45 & 50 & 0.90 & 1820 & 38 \\
0.78 & 0.021 & 0.093 & 8160 & 5.0 & 20 & 0.43 & 40 & 0.86 & 1600 & 40 \\
0.72 & 0.025 & 0.106 & 9300 & 3.7 & 17 & 0.43 & 35 & 0.88 & 1600 & 50 \\
0.60 & 0.036 & 0.088 & 7720 & 3.0 & 10 & 0.36 & 25 & 0.94 & 1240 & 50 \\
0.72 & 0.025 & 0.133 & 11,670 & 3.0 & 10 & 0.25 & 30 & 0.75 & 1490 & 63 \\
0.50 & 0.052 & 0.133 & 11,670 & 1.4 & 10 & 0.52 & 18 & 0.94 & 1030 & 90 \\
0.50 & 0.052 & 0.192 & 16,840 & 1.0 & - & - & 12 & 0.624 & 1030 & 130 \\
\hline
\end{tabular}

Table 1: Summary of the approximate initial conditions, dimensionless parameters and measured time scales in the experiments.

Figure 14 gives the dependence of the linear-non-linear transition times on the initial interaction parameter. The experimental data points are compared with the curve $(t / \tau)_{l}=0.8 N_{0}^{2}$. Though an $N_{0}^{2}$-law for the transition time looks plausible, transition data at higher values of $N_{0}$ are needed to validate the theoretical prediction.

In all experiments (with the possible exception of the case $N_{0}=1$ ), the correlation coefficient of the voltages measured at the opposite walls of the test section rises to a value less than unity. This implies that, even if the swirl motion has diffused as far as the other wall of the cavity, the two potentials may not be equal. Earlier studies on the two-dimensionality of MHD flows reported by Votsish and Kolesnikov (1975) and Branover (1978) have indicated that the correlation coefficients of the velocity fluctuations at two points aligned with the magnetic field, but spaced apart never became equal to unity, even when the field was strong. However, at the end of our measured non-linear decay phase, we find that the velocity of the vortex has fallen by one order of magnitude $\left(u \sim 0.01 \mathrm{~m} \mathrm{~s}^{-1}\right)$. When the value of the potential difference approaches the limit of resolution $(\sim 10 \mu \mathrm{V})$, it is likely that the recorded correlation coefficients are prone to errors. 


\subsection{Attempted experiments at low $N_{0}$}

Experiments at interaction parameters smaller than unity have not yielded conclusive results. A $t^{-9 / 5}$ decay law has been found until $t \approx 10 \tau$, when $N_{0}=0.9$. At low $N_{0}$, the evolution of a vortex is complex. The turn-over time of the vortex is sufficiently smaller than the Joule time, so that the dominant inertial forces cause

the vortex to break down into a ring that propagates radially outward. On the other hand, angular momentum also diffuses along the field lines due to weak Joule dissipation. It is not clear whether, in our experiments, wall friction damps out the radial motion of the structure. An experiment at $N_{0}=0.7$ gave a $t^{-1.5}$ decay law for square of the velocity (figure 15), which cannot be explained based on our model for non-linear evolution. There is still a well-defined rise in the correlation coefficient. Experiments at smaller $N_{0}$ are beset with further uncertainties. If the magnetic field is kept strong enough, the initial velocity must be high enough to keep $N_{0}$ small. This results in a value of $R e_{H a} \sim 200$, which could mean that the Hartmann layer is turbulent, and the dissipation rate there is higher; see, for example, Lingwood and Alboussière (1999). All the experiments referred to in table 1 have been performed at $R e_{H a} \leq 130$. Experiments at higher values of $R e_{H a}$ have not given consistent decay laws. For a weaker field $(B=0.3 \mathrm{~T})$, it is not clear why consistent results are not obtained, though $R e_{H a}$ is small enough to give a sound result. A possible reason could be the higher contribution of Hartmann friction to the overall damping of the vortex; see Appendix A.2. As noted by the authors (Sreenivasan and Alboussière, 2000 ), the evolution of a vortex at low interaction parameters is complex. The turn-over time of the vortex is sufficiently smaller than the Joule time, so that the dominant inertial forces cause the vortex to break down into a ring that propagates radially outward. On the other hand, the weak Lorentz forces cause the angular momentum to diffuse along the field lines.

The study of the growth of length scales using the internal side probes has been unsuccessful because of the intrusive nature of the measurement system. It was 
hoped that, by positioning these probes outside the intense swirl region of the vortex, the correlations of the velocity measured by them could be studied. The voltages measured were too low to give any conclusive result.

\section{Discussion}

In this paper, we have described an experimental study that investigates the evolution of a vortex in a liquid metal subject to a uniform magnetic field. The initial interaction parameter is varied to study how the relative magnitudes of the electromagnetic and non-linear inertial forces affect the decay of the vortex. The present study is unique in two respects:

1. The initial "linear" phase of decay of an MHD flow field at large $N_{0}$, has been observed for the first time in a laboratory experiment. This regime, modelled theoretically by Moffatt in 1967 and by subsequent researchers, can be obtained in a MHD experiment only if the magnetic field is sufficiently strong or the initial velocity is sufficiently small, both of which contribute to a high value of $N_{0}$.

2. It has been possible to demonstrate experimentally that, for large $N_{0}$, there exist two different regimes of decay for a three-dimensional vortex evolving in a large domain. This interesting behaviour was predicted by Sreenivasan and Alboussière, based on the definition of the true interaction parameter, $N_{t}$. This dimensionless number decreases and tends to a constant of order one at the end of the linear phase, and remains invariant during the subsequent non-linear evolution.

As the initial interaction parameter is lowered from a high value, the linear phase of evolution gets shorter, and when $N_{0}=1$, it disappears totally. Though the definition of $N_{0}$ is based on an order of magnitude of the initial velocity estimated by the 
relation (16), the particular result for the case $N_{0}=1$ shows that the calculated value can be confidently accepted as the actual value realized in the experiment.

Though the method of estimating velocities by wall potential measurements is only approximate unless the flow is fully two-dimensional, the experimental decay laws have agreed fairly well with the order-of-magnitude estimates. This indicates that (a) except for the first few Joule times, the current density in the core of the flow can be considered to be small, and (b) Hartmann layer dissipation of the flow is small in comparison with the ohmic dissipation in the core, for the field strengths considered in the present study. From a theoretical point of view, our results are consistent with $(a)$ the component of angular momentum along the axis of the vortex being nearly conserved, $(b)$ viscous damping of the vortex being negligible in comparison with ohmic damping in both regimes of evolution and $(c)$ the actual ratio of the Lorentz to the non-linear inertial forces being approximately a constant during the non-linear phase. Of the above, the angular momentum appears to be a robust invariant during the duration of an experiment (see Appendix A.1).

The laws of decay of a vortex were derived assuming that the structure was free from the influence of boundaries. It would be relatively difficult to create and study a swirling vortex in the core of the test section by non-intrusive means. The effect of the wall on the initial evolution of the vortex is not well understood. If the good agreement of the experimental results with the theoretical estimates for the two regimes $\left(\overline{u^{2}} \sim(t / \tau)^{-1}, \sim(t / \tau)^{-9 / 5}\right)$ is any indication, our initial assumption that the wall does not play a significant role in the long-time evolution of the vortex (until a two-dimensional state is reached) was well-founded.

The use of potential measurements at the wall to study core flow dynamics has the advantage that the system is non-intrusive, but velocities obtained are those just outside the Hartmann layer where the vortex is generated. At the propagating front of the vortex, the velocity will be lower. However, the rate of decay of $u^{2}$ at every section follows the same laws, so that the "local" measurements obtained near 
one wall do give a "global" picture of the evolution. An experimental study of the growth of the length scales, $l_{\|}$and $l_{\perp}$, though difficult to perform, should give a more complete description of the decay, especially in the non-linear phase. In the absence of these data, one needs to have recourse to the theoretical laws of evolution of the length scales, and adopt the relation (13) for interpreting the experimental results.

In principle, $N_{0}$ can be varied by adjusting the magnetic flux density as well as the initial swirl velocity of the vortex. However, we have found that a minimum initial velocity of about $0.1 \mathrm{~m} \mathrm{~s}^{-1}$, as estimated by the relation (16), needs to be ensured so that the velocity is within measurable limits throughout the duration of an experiment. Hence, higher values of $N_{0}(>5)$ have been achieved by increasing the magnetic flux density, and not by reducing the the intensity of the pulsed current. The maximum value of $N_{0}$ realized in this study $(\approx 8)$ is thus limited by the maximum field strength. If sufficiently higher fields can be imposed, a purely-linear evolution can be seen until two-dimensionality is reached. Further, the range of observation of the power laws for the linear and non-linear phases is limited by the maximum magnetic field available as well as the finite size of the cavity. A higher magnetic field and a larger transverse dimension of the box should permit the study of one decade of decay of the kinetic energy in both phases. Despite the above physical constraints in the experiment, a clear transition from one regime to another can be captured during the vortex evolution, by choosing a suitably-high value of the initial interaction parameter.

It would be interesting to consider the implication of our results in the larger context of low $R_{m}$ MHD turbulence. It is noteworthy that the $t^{-1.7}$ decay law for the energy, $\overline{u^{2}}$, obtained by Alemany et al. was for $N_{0} \sim 1$. In a grid-generated turbulence experiment like theirs, it would have been difficult to achieve high values of $N_{0}$ because of the modest magnetic field strength and the small initial length scale. Hence, the regime observed by them was non-linear from the beginning. In the absence of published experimental data on the long-time evolution of MHD turbulence at large 
$N_{0}$, it is reasonable to suppose that energy is dissipated as $(t / \tau)^{-1 / 2}$ during a linear phase $\tau<t<N_{0}^{4 / 3} \tau$, and then, as $(t / \tau)^{-1.7}$ during the subsequent non-linear phase.

\section{A Appendix}

\section{A.1 Decay of angular momentum}

We have seen that conservation of the component of angular momentum parallel to the external magnetic field is an important condition that governs the evolution of a vortex in an infinite domain. However, in our experiment, vortices are generated at one of the vertical walls of the test section. As the flow structure elongates, viscous shear at the wall where the structure is generated opposes the swirl motion and causes the angular momentum to decay in time. The magnitude of the shear stress is

$$
\begin{aligned}
\tau_{H} & =\mu \frac{\partial u_{H}}{\partial y}=\mu \frac{\partial}{\partial y}\left[u_{c}\left(1-e^{-\eta H a}\right)\right] \\
& =\mu u_{c} e^{-\eta H a}(H a / L),
\end{aligned}
$$

where $u_{H}$ is the velocity in the Hartmann layer, which is known to vary exponentially along the co-ordinate parallel to the magnetic field, $y$ (Moreau, 1990). $\eta$ is the dimensionless length $y / L$, and $H a$ the Hartmann number. As $\eta$ increases, the velocity approaches the value in the core, $u_{c}$. The shear stress at the wall $(y=0)$ is

$$
\tau_{H, 0}=\mu u_{c}(H a / L) .
$$

and the torque acting on the vortex due to shear at one wall is given by

$$
T_{H}=\int_{r=0}^{\delta} \mu u_{c} \frac{H a}{L} r 2 \pi r d r \sim \frac{\mu u_{c} H a \delta^{3}}{L},
$$

where $\delta$ is the characteristic radius of the vortex. Now the angular momentum is defined by

$$
\begin{aligned}
H & =\rho \int_{V}(\mathbf{x} \times \mathbf{u}) d V=\rho \int_{r=0}^{\delta} u_{c} r 2 \pi r d r L \\
& \sim \rho u_{c} \delta^{3} L,
\end{aligned}
$$


where $\rho$ is the density of the liquid and $L$ the maximum length scale attained by the vortex. Since $\mathrm{d} H / \mathrm{d} t=-T_{H}$, the characteristic time for decay of $H$ is

$$
t_{H}=\frac{L^{2}}{\nu H a} \sim\left(\frac{\rho}{\sigma \nu}\right)^{1 / 2} \frac{L}{B}
$$

where $B$ is the flux density. For a field strength of 0.5 Tesla acting on mercury $\left(\nu=1.144 \times 10^{-7} \mathrm{~m}^{2} \mathrm{~s}^{-1}, \sigma=1.04 \times 10^{6} \Omega^{-1} \mathrm{~m}^{-1}, \rho=13.55 \times 10^{3} \mathrm{~kg} \mathrm{~m}^{-3}\right)$, contained in the chamber used in our experiments (where $L=80 \mathrm{~mm}$ ), the above time scale is found to be approximately 50 seconds. Since this time scale is much greater than the typical duration of one experiment ( $\sim 1$ second), it can be stated with confidence that the angular momentum of the vortex is nearly invariant during the measured evolution.

The time scale for decay of $H$ is found to be equal to the time for the decay of turbulence due to Hartmann braking (Joule damping in the Hartmann layers) estimated by Sommeria and Moreau (1982).

\section{A.2 Hartmann layer dissipation vs. core dissipation}

The energy decay of a vortex in a magnetic field is modelled theoretically under the assumption that the domain is infinite, or the electrically-insulating boundaries are situated far enough from the vortex, so that their influence is not felt in the evolution process. Hence, the decay is solely due to Joule dissipation in the core of the flow. In a real life experiment, the presence of boundaries normal to the magnetic field give rise to Hartmann layers where the velocity goes to zero at the walls. Viscous and Joule dissipation in the Hartmann layers also contribute to the destruction of kinetic energy. It is instructive to evaluate the relative magnitudes of the core and Hartmann layer dissipation rates in the present experiment.

The viscous dissipation per unit volume in the Hartmann layer is

$$
D_{v}=\mu\left(\frac{\partial u_{H}}{\partial y}\right)^{2}=\sigma B^{2} u_{c}^{2} e^{-2 \eta H a} .
$$

Here, we have made use of the exponential solution for $u_{H}$, which goes to zero at the 
wall $(y=0)$ and tends to the core velocity, $u_{c}$ at large $\eta$. The Joule dissipation in the Hartmann layer can be estimated from the current flowing in the layer. Since the gradient of potential remains practically unchanged through the Hartmann layer, the current is given by the expressions

$$
\begin{aligned}
\mathbf{j}_{H} & =\sigma\left[-(\nabla \phi)_{H a}+(\mathbf{u} \times \mathbf{B})_{H a}\right], \\
j_{H} & =\sigma\left[-u_{c} B+u_{c}\left(1-e^{-\eta H a}\right) B\right], \\
& =-\sigma B u_{c} e^{-\eta H a} .
\end{aligned}
$$

The Joule dissipation per unit volume is then given by

$$
D_{j}=\mathbf{j}^{2} / \sigma=\sigma B^{2} u_{c}^{2} e^{-2 \eta H a}
$$

The magnitudes of the viscous and Joule dissipation rates in the Hartmann layer are equal. The total dissipation per unit mass can be estimated as

$$
\begin{aligned}
D_{H} & \sim 2 \frac{\sigma B^{2}}{\rho} u_{c}^{2} \int_{y=0}^{\infty} e^{-2 \eta \frac{y}{L} H a} \delta^{2} d y \\
& \sim \frac{u_{c}^{2} \delta^{2} L}{\tau H a}
\end{aligned}
$$

where $\delta$ is the radius of the eddy.

The dissipation in the core of the flow, as estimated in (10), is

$$
D_{c} \sim-\frac{E}{\tau}\left(\frac{l_{\perp}}{l_{\|}}\right)^{2} \sim-\frac{u_{c}^{2} \delta^{2} l_{\|}}{\tau} \frac{\delta^{2}}{l_{\|}^{2}} .
$$

The ratio of the Hartmann dissipation to the core dissipation of a vortex will be

$$
\frac{D_{H}}{D_{c}}=\frac{1}{H a} \frac{l_{\|} L}{\delta^{2}}
$$

For a given magnetic field, Hartmann dissipation will be the most significant when the parallel length scale of the eddy attains the size of the cavity $\left(l_{\|} \approx L\right)$. If the eddy is fully two-dimensional, Hartmann layers at both walls contribute to the damping of the vortex, but the current in the core of the flow would have dropped to zero. For a field strength of $0.5 \mathrm{~T}$ acting on a structure of radius $5 \mathrm{~mm}$ and length nearly 
$40 \mathrm{~mm}$ in mercury, the above ratio will be 0.12 . Lower the field strength, larger the contribution of Hartmann dissipation to the overall damping. For a field strength of $0.1 \mathrm{~T}$, the Hartmann dissipation can account for up to $40 \%$ of the total dissipation or more depending on the size of the vortex. To ensure that the measured decay of kinetic energy is a true representation of the dissipation in the core, the applied magnetic field should be reasonably high, so that the ratio (37) is small.

\section{Acknowledgements}

Binod Sreenivasan's work was supported by a Cambridge Nehru-Chevening scholarship and a Junior Research Fellowship from Hughes Hall, Cambridge. 


\section{References}

H. Branover, Yu.M. Gel'fgat, L.G. Kit, and A.B. Tsinober. Study of MHD turbulence in tubes using conduction anemometers. Mekhanika Zhidkosti i Gaza, 5(2): 35-44, 1970a.

N.M. Slyusarev. Effect of a transverse magnetic field on turbulent channel flows. Magnitnaya Gidrodinamika, 7(1):15-20, 1971.

O. Lielausis. Liquid metal magnetohydrodynamics. Atomic Energy Review, 13: $527-581,1975$.

Yu.B. Kolesnikov and A.B. Tsinober. Two-dimensional turbulent flow behind a circular cylinder. Magnitnaya Gidrodinamika, 8(3):23-31, 1972.

A.D. Votsish and Yu.B. Kolesnikov. Spatial correlation and vorticity in twodimensional homogeneous turbulence. Magnitnaya Gidrodinamika, 11(3):25-28, 1975.

J. Sommeria. Electrically driven vortices in a strong magnetic field. J. Fluid Mech., 189:553-569, 1988.

T. Alboussière, V. Uspenski, and R. Moreau. Quasi-2d MHD turbulent shear layers. Experimental Thermal and Fluid Science., 20:19-24, 1999.

A. Alemany, R. Moreau, P.L. Sulem, and U. Frisch. Influence of an external magnetic field on homogeneous MHD turbulence. J. Méc, 18:277-313, 1979.

Ph. Caperan and A. Alemany. Turbulence homogène MHD à faible nombre de Reynolds magnetique. Etude de la transition vers la phase quasi bidimensionnelle et caractérisation de son anisotropie. J. Méc. Théor. Appl., 4:175-200, 1985.

H.K. Moffatt. On the suppression of turbulence by a uniform magnetic field. $J$. Fluid Mech., 28:571-592, 1967.

J. Sommeria and R. Moreau. Why, how and when, MHD turbulence becomes twodimensional. J. Fluid Mech., 118:507-518, 1982. 
P.A. Davidson. Magnetic damping of jets and vortices. J. Fluid Mech., 299:153-186, 1995.

B. Lehnert. On the behaviour of an electrically-conducting liquid in a magnetic field. Arkiv For Fysik, 5(5), 1952.

R. Moreau. Magnetohydrodynamics. Kluwer Acad. Publ., 1990.

B. Sreenivasan and T. Alboussière. Evolution of a vortex in a magnetic field. Eur.J.Mech. B-Fluids., 19:403-421, 2000.

I.A. Platnieks and Ya.Zh. Freiberg. Turbulence and some problems in the stability of flows with M-shaped velocity profiles. Magnitnaya Gidrodinamika, 7(2):29-34, 1971.

P. Tabeling, S. Burkhart, O. Cardoso, and H. Willaime. Experimental study of freely-decaying two-dimensional turbulence. Phys. Rev. Lett., 67:3772-3774, 1991.

H. Branover, Yu.M. Gel'fgat, L.G. Kit, and I.A. Platnieks. Effect of a transverse magnetic field on the intensity profiles of turbulent velocity fluctuations in a channel of rectangular cross-section. Magnitnaya Gidrodinamika, 6(3):41-50, 1970b.

L.G. Kit. Turbulent velocity fluctuation measurements using a conduction anemometer with a three-electrode probe. Magnitnaya Gidrodinamika, 6(4):41-46, 1970.

I.A. Platnieks. Comparison of the hot-wire anemometric and conduction methods of measuring velocity characteristics of a flow of mercury in a transverse magnetic field. Magnitnaya Gidrodinamika, 3:140-142, 1971.

B. Sreenivasan. Evolution of inertial flow structures in the presence of a magnetic field. PhD thesis, Department of Engineering, University of Cambridge, 2000.

U. Burr, L. Barleon, U. Muller, and A. Tsinober. Turbulent transport of momentum and heat in magnetohydrodynamic rectangular duct flow with strong sidewall jets. J. Fluid Mech., 406:247-279, 2000. 
H. Branover. Magnetohydrodynamic Flows in Ducts. Halsted Press, 1978.

R.J. Lingwood and T. Alboussière. On the stability of the Hartmann layer. Phys. Fluids, 11(8):19-24, 1999. 


\section{Figure captions}

Figure 1. Evolution of a spherical vortex whose axis is parallel to an external field, at large $N_{0}$. The vortex elongates into a columnar structure. $l_{\|}$and $l_{\perp}$ are the typical longitudinal and transverse dimensions of the vortex. $t_{2}>t_{1}>0$.

Figure 2. The experimental cell showing 1. Plexiglas test section. 2. Plexiglas base. 3. O-ring seal. 4. Hollow stainless steel shaft. 5. Side potential probes. 6. Expansion chamber. 7. Argon circulation port. 8. Electrode array for current injection and wall-potential probes.

Figure 3. Direction of the injected current from the central electrode to the surrounding electrodes, and the resultant velocity generated in the mercury. The location of the potential probes is also shown.

Figure 4. Data (in $\mathrm{mV}$ ) recorded during one realization, after low-pass digital filtering, for $N_{0}=3$. The initial spike corresponds to the current injection.

Figure 5. Mean square value of the measured potential difference, normalized with respect to the value at $t=\tau$, and magnified in the range $2<t / \tau<10$. Averaging over 5,10 and 20 realizations gives nearly the same result, indicating a "deterministic" behaviour.

Figure 6. Mean square value of the measured potential difference, normalized with respect to the value at $t=\tau$, and magnified in the range $10<t / \tau<60$. The first curve from bottom is the one obtained for 5 realizations. The curve obtained from averaging over 20 realizations has the lowest amplitude of fluctuations.

Figure 7. (a) Mean square velocity of a vortex as a function of time, for $N_{0}=3$, and the corresponding velocity correlation coefficients shown superposed. $u_{1}$ and $u_{2}$ are the velocities measured on opposite walls normal to the external field, at points that lie along the field lines. They are derived from the respective potential differences. The magnetic field, $B=0.6 T$. 
Figure 8. Mean square velocity of a vortex as a function of time, for $N_{0}=3$, and the corresponding velocity correlation coefficients. $B=0.72 T$.

Figure 9. Mean square velocity, for $N_{0}=3.7$, and the corresponding correlation coefficients. $B=0.72 \mathrm{~T}$.

Figure 10. Mean square velocity of a vortex, for $N_{0}=6$, and the corresponding velocity correlation coefficients. $B=0.88 T$.

Figure 11. Mean square velocity of a vortex, for $N_{0}=7.7$, and the corresponding velocity correlation coefficients. $B=0.92 T$.

Figure 12. Mean square velocity of a vortex as a function of time, for $N_{0}=1.4$, and the corresponding velocity correlation coefficients. $B=0.50 T$.

Figure 13. Mean square velocity of a vortex, for $N_{0}=1.0$, and the corresponding velocity correlation coefficients. $B=0.50 T$. The absence of the linear phase may be noted.

Figure 14. Variation of the linear-non-linear transition time with $N_{0}$, in logarithmic co-ordinates. The experimental data points are shown by the symbol $*$, and the solid line is the curve $(t / \tau)_{l}=0.8 N_{0}^{2}$.

Figure 15. Mean square velocity for $N_{0}=0.7$, and the corresponding correlation coefficients. $B=0.42 \mathrm{~T}$. 


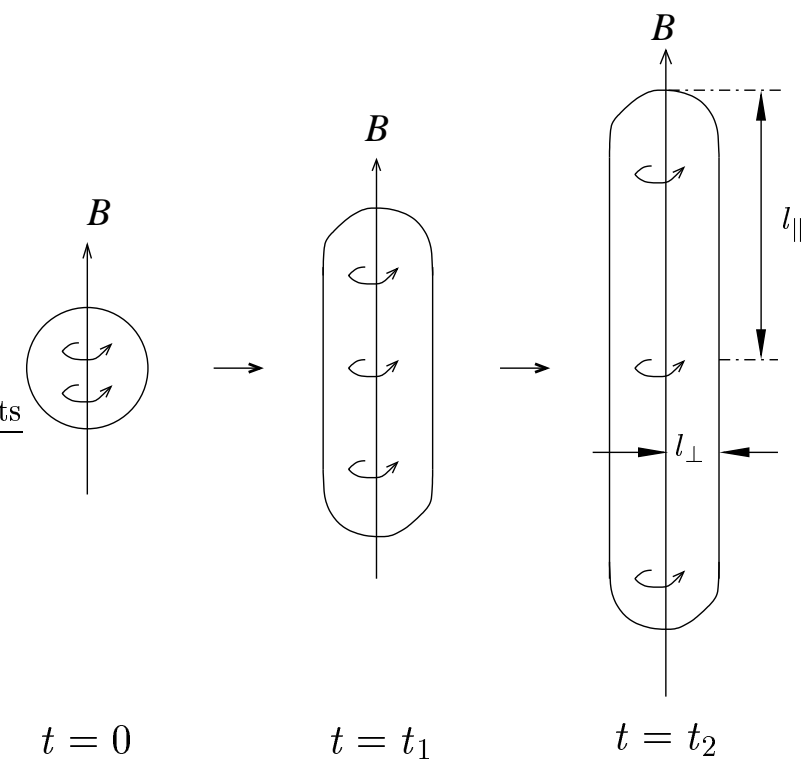

Figure 1: Evolution of a spherical vortex whose axis is parallel to an external field, at large $N_{0}$. The vortex elongates into a columnar structure. $l_{\|}$and $l_{\perp}$ are the typical longitudinal and transverse dimensions of the vortex. $t_{2}>t_{1}>0$. 


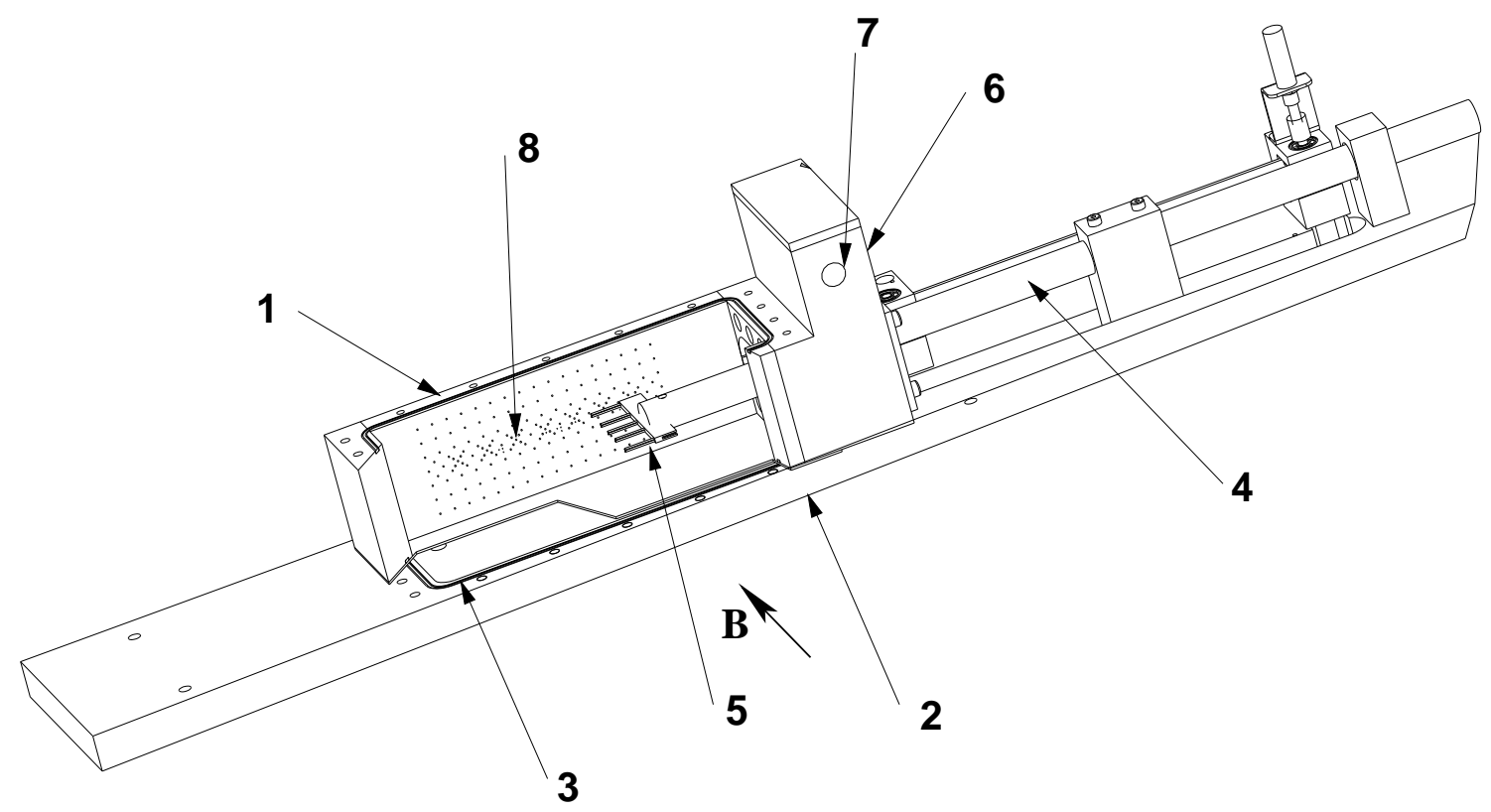

Figure 2: The experimental cell showing 1. Plexiglas test section. 2. Plexiglas base. 3. O-ring seal. 4. Hollow stainless steel shaft. 5. Side potential probes. 6. Expansion chamber. 7. Argon circulation port. 8. Electrode array for current injection and wallpotential probes. 

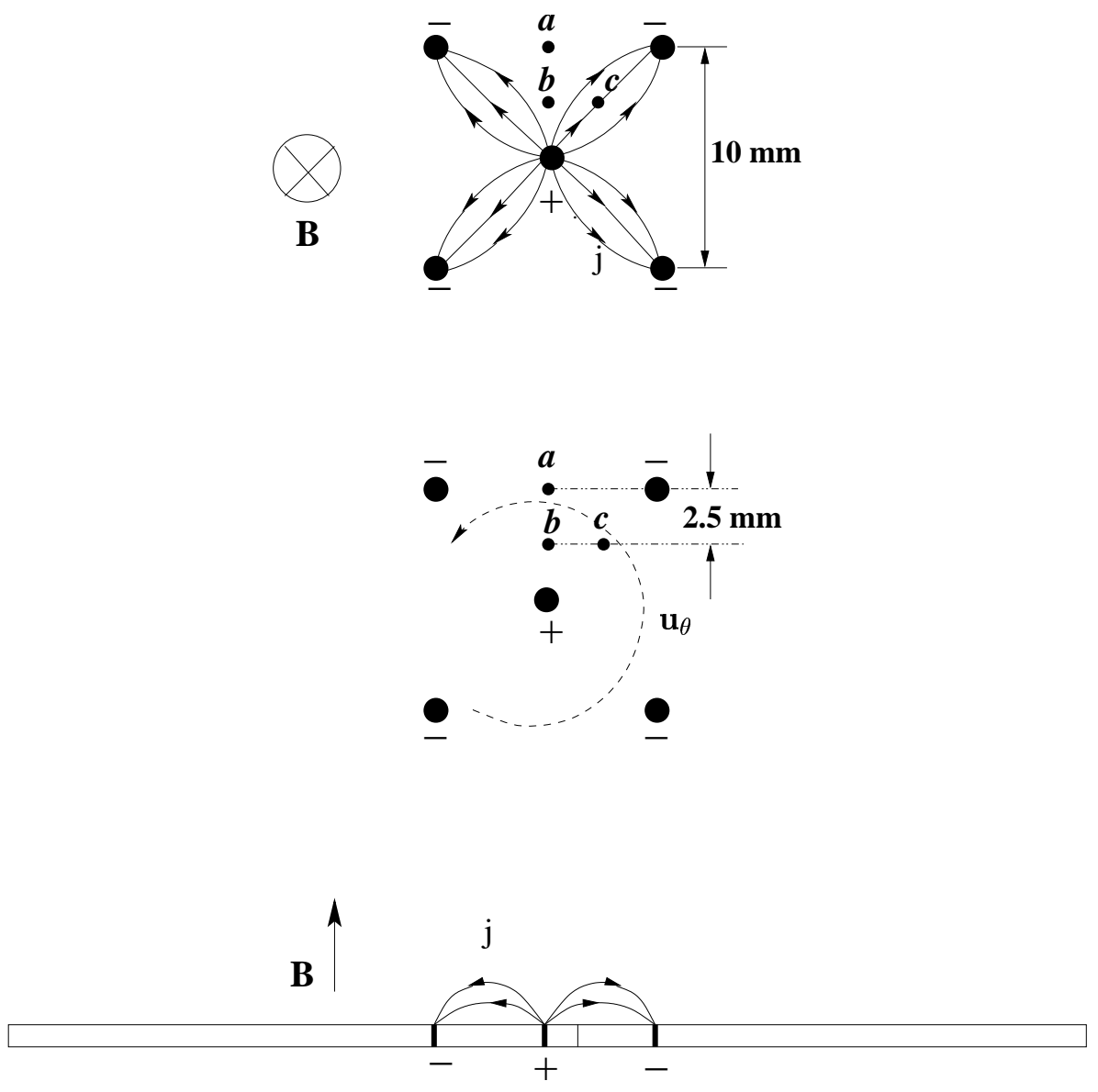

- Wall potential probes (on both vertical walls)

- Current injection electrodes

Figure 3: Direction of the injected current from the central electrode to the surrounding electrodes, and the resultant velocity generated in the mercury. The location of the potential probes is also shown. 


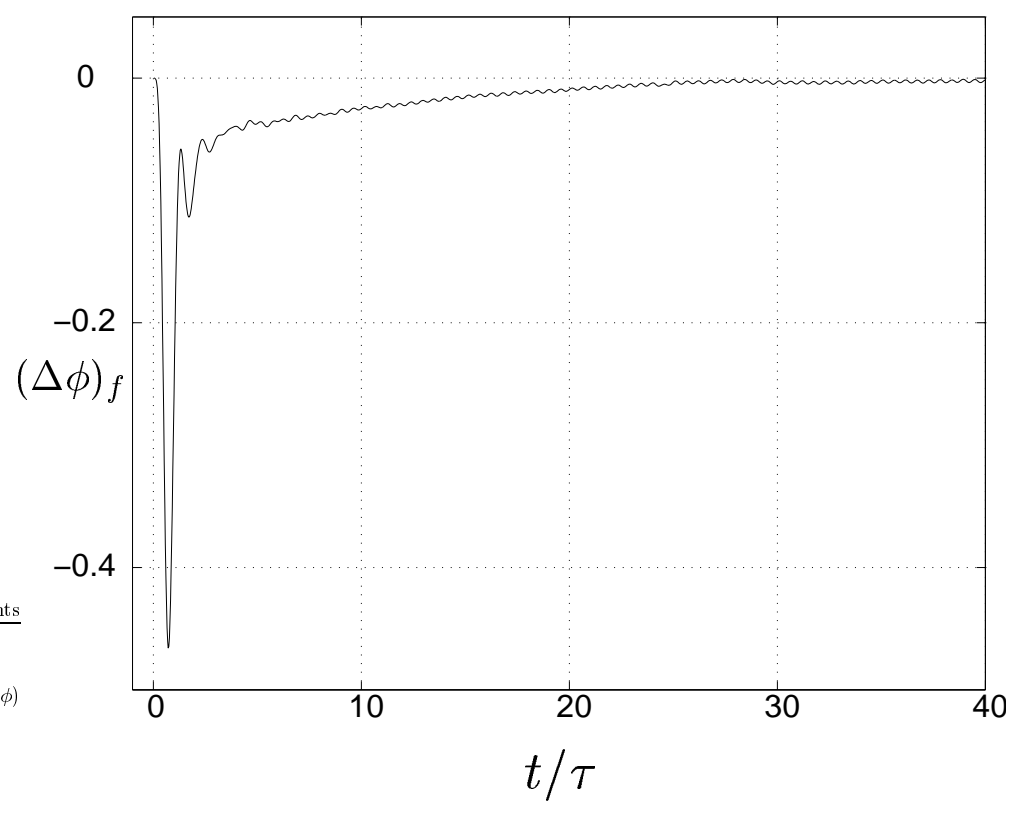

Figure 4: Data (in $\mathrm{mV}$ ) recorded during one realization, after low-pass digital filtering, for $N_{0}=3$. The initial spike corresponds to the current injection. 


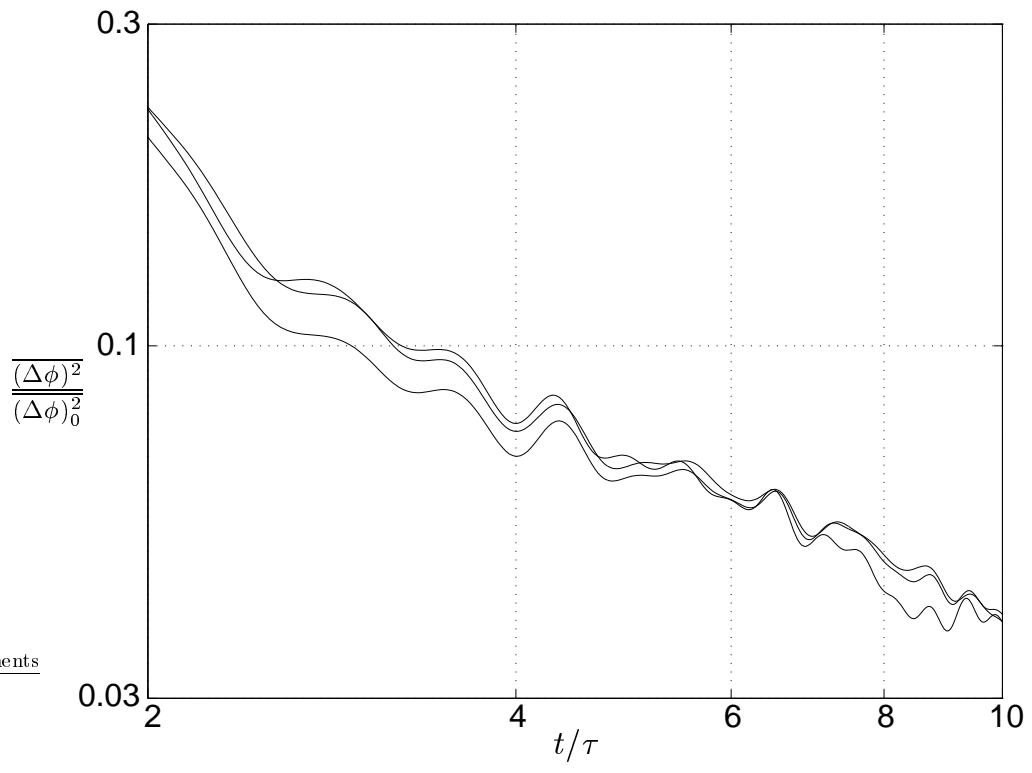

Figure 5: Mean square value of the measured potential difference, normalized with respect to the value at $t=\tau$, and magnified in the range $2<t / \tau<10$. Averaging over 5,10 and 20 realizations gives nearly the same result, indicating a "deterministic" behaviour. 


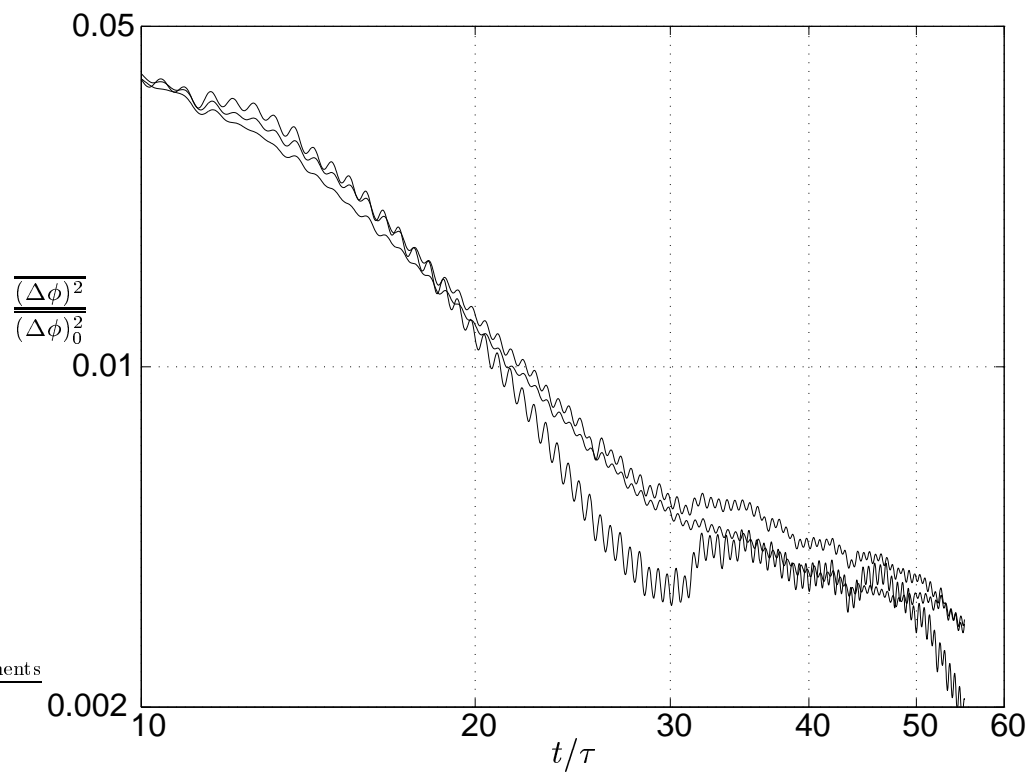

Figure 6: Mean square value of the measured potential difference, normalized with respect to the value at $t=\tau$, and magnified in the range $10<t / \tau<60$. The first curve from bottom is the one obtained for 5 realizations. The curve obtained from averaging over 20 realizations has the lowest amplitude of fluctuations. 


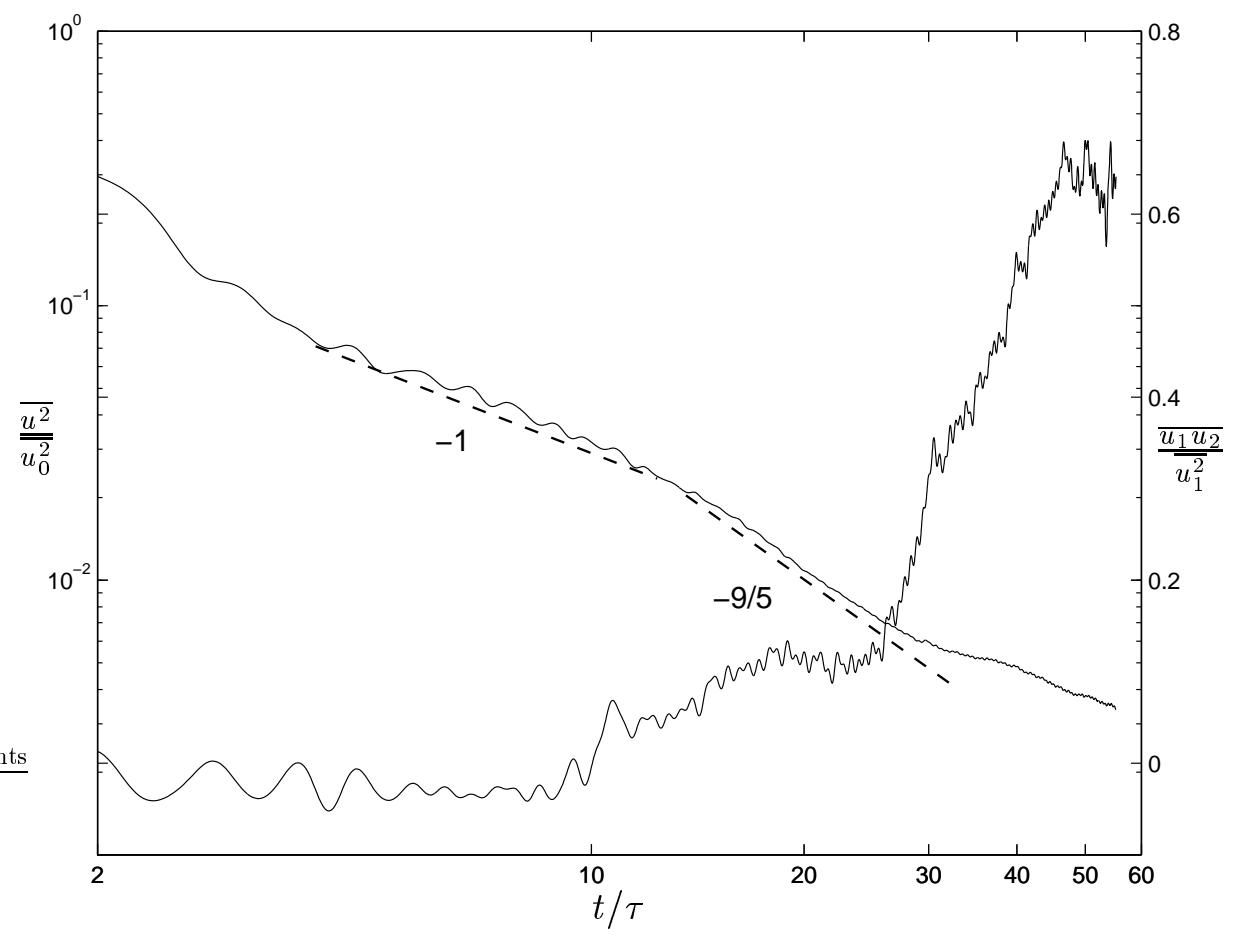

Figure 7: (a) Mean square velocity of a vortex as a function of time, for $N_{0}=3$, and ( $b$ the corresponding velocity correlation coefficients. $u_{1}$ and $u_{2}$ are the velocities measured on opposite walls normal to the external field, at points that lie along the field lines. They are derived from the respective potential differences. The magnetic field, $B=0.6 T$. 

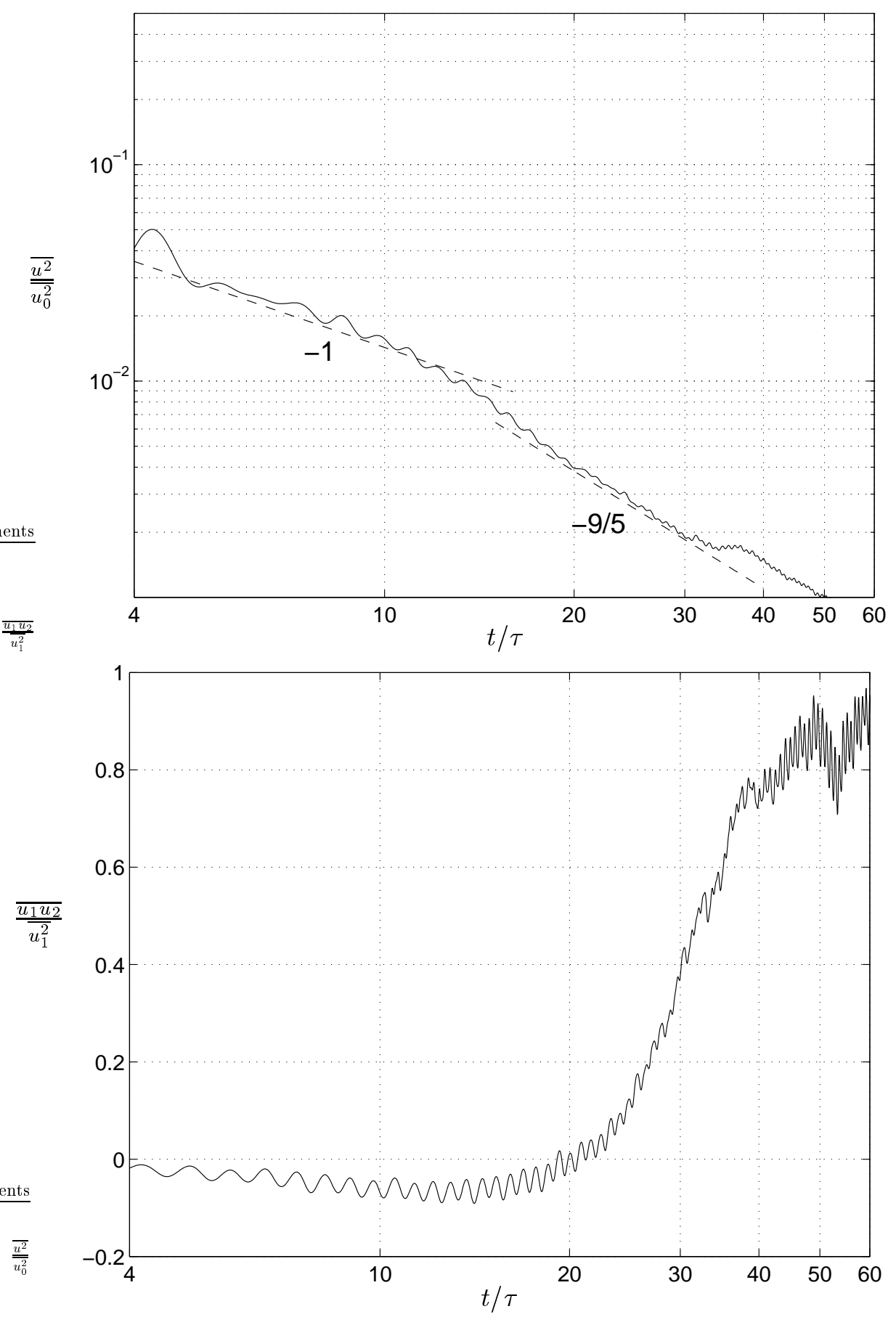

Figure 8: Mean square velocity of a vortex as a function of time, for $N_{0}=3$, and the corresponding velocity correlation coefficients. $B=0.72 T$. 


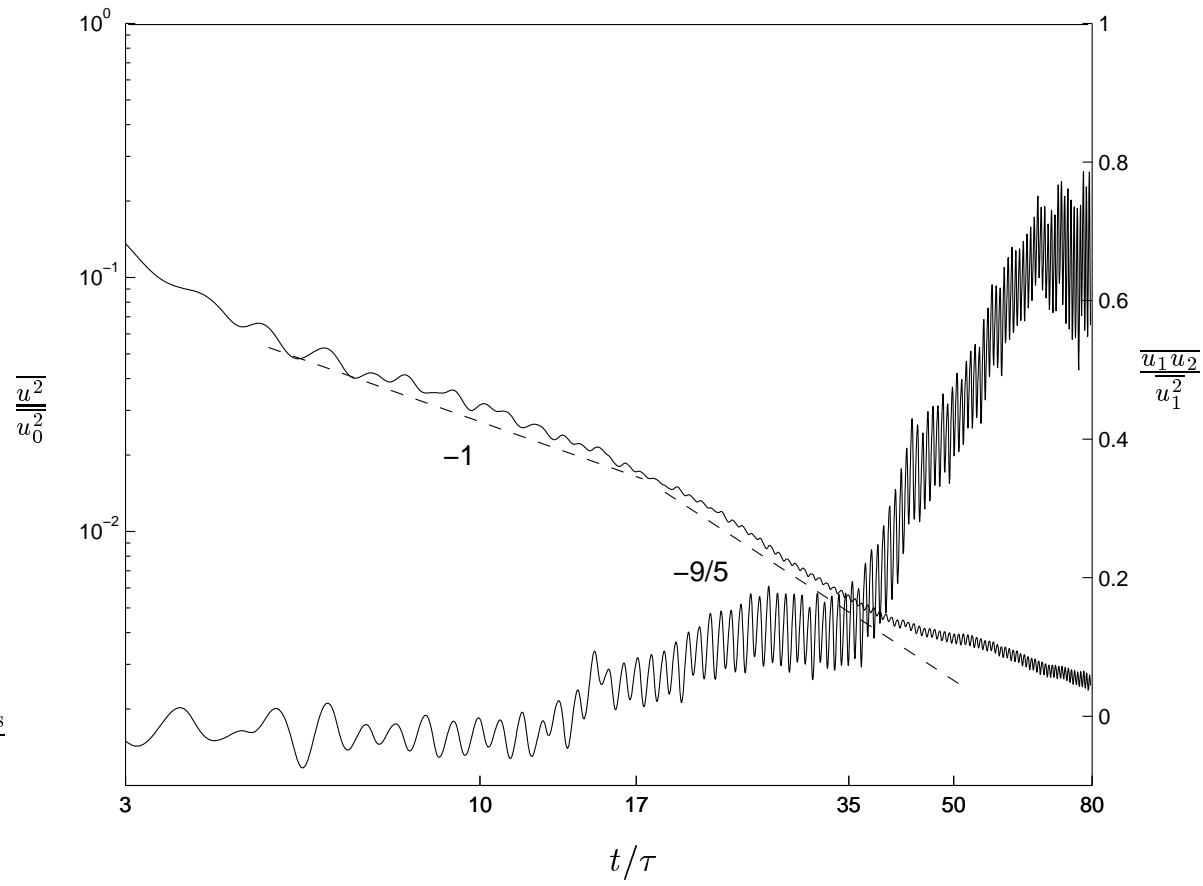

Figure 9: Mean square velocity, for $N_{0}=3.7$, and the corresponding correlation coefficients. $B=0.72 \mathrm{~T}$ 


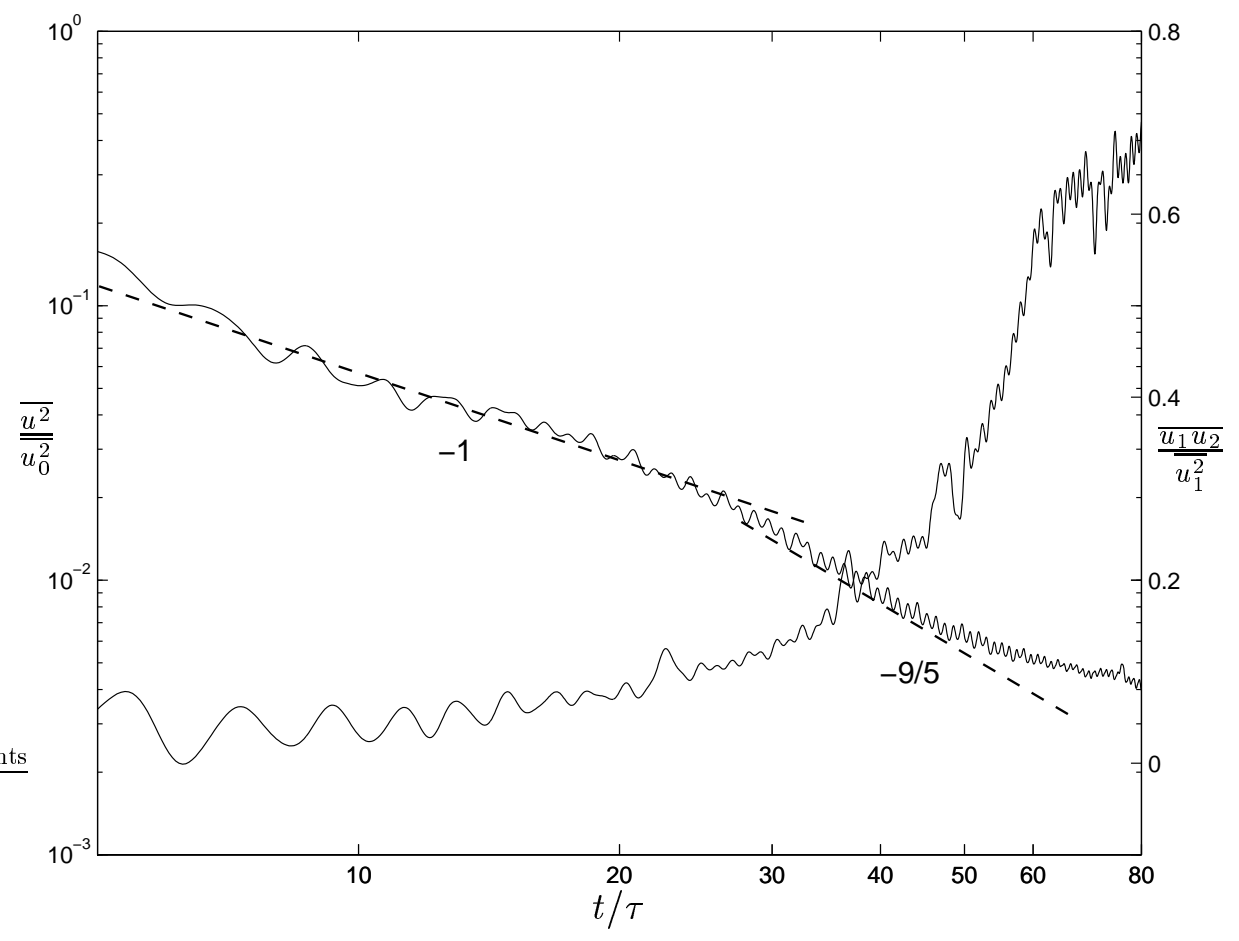

Figure 10: Mean square velocity of a vortex, for $N_{0}=6$, and the corresponding velocity correlation coefficients. $B=0.88 T$. 

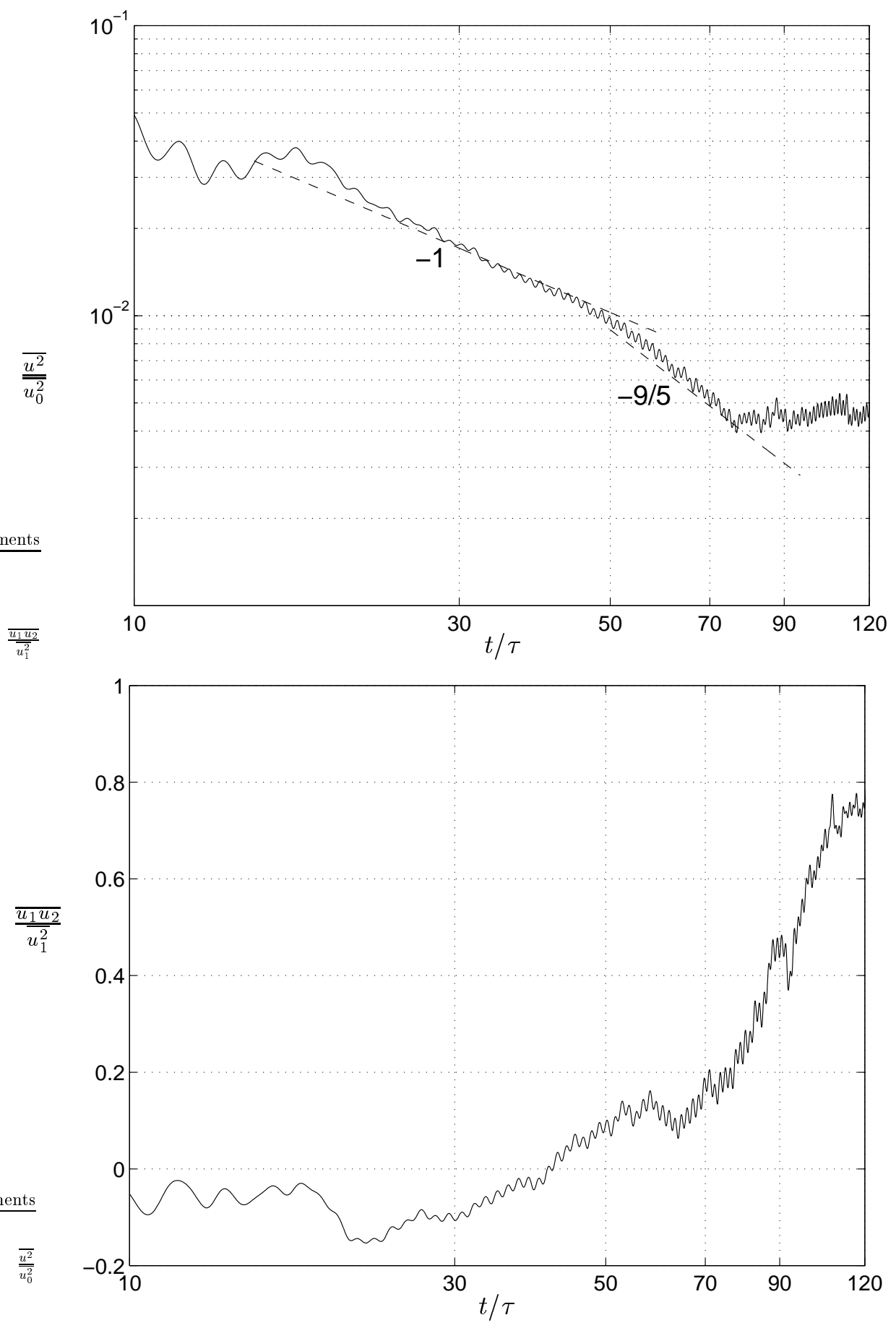

Figure 11: Mean square velocity of a vortex, for $N_{0}=7.7$, and the corresponding velocity correlation coefficients. $B=0.92 T$. 


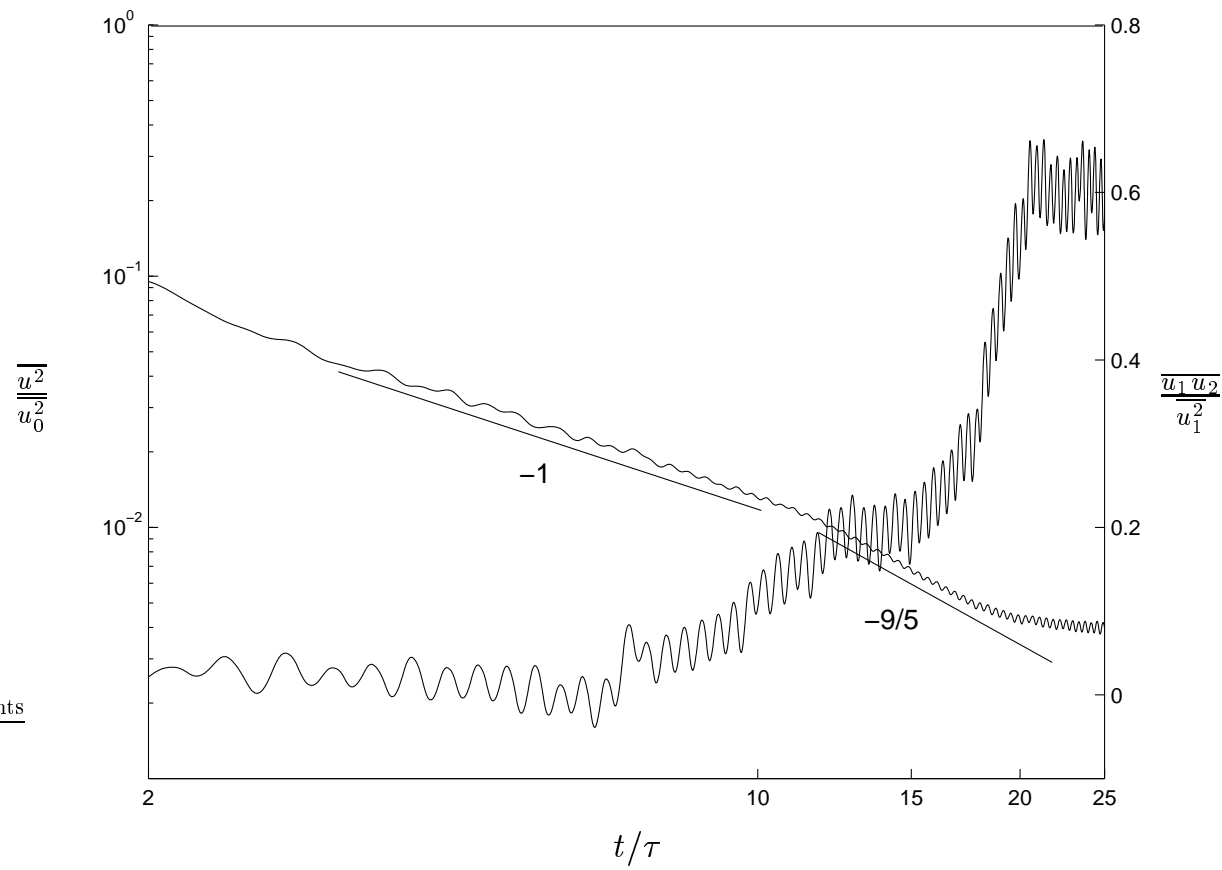

Figure 12: Mean square velocity of a vortex as a function of time, for $N_{0}=1.4$, and the corresponding velocity correlation coefficients. $B=0.50 T$. 


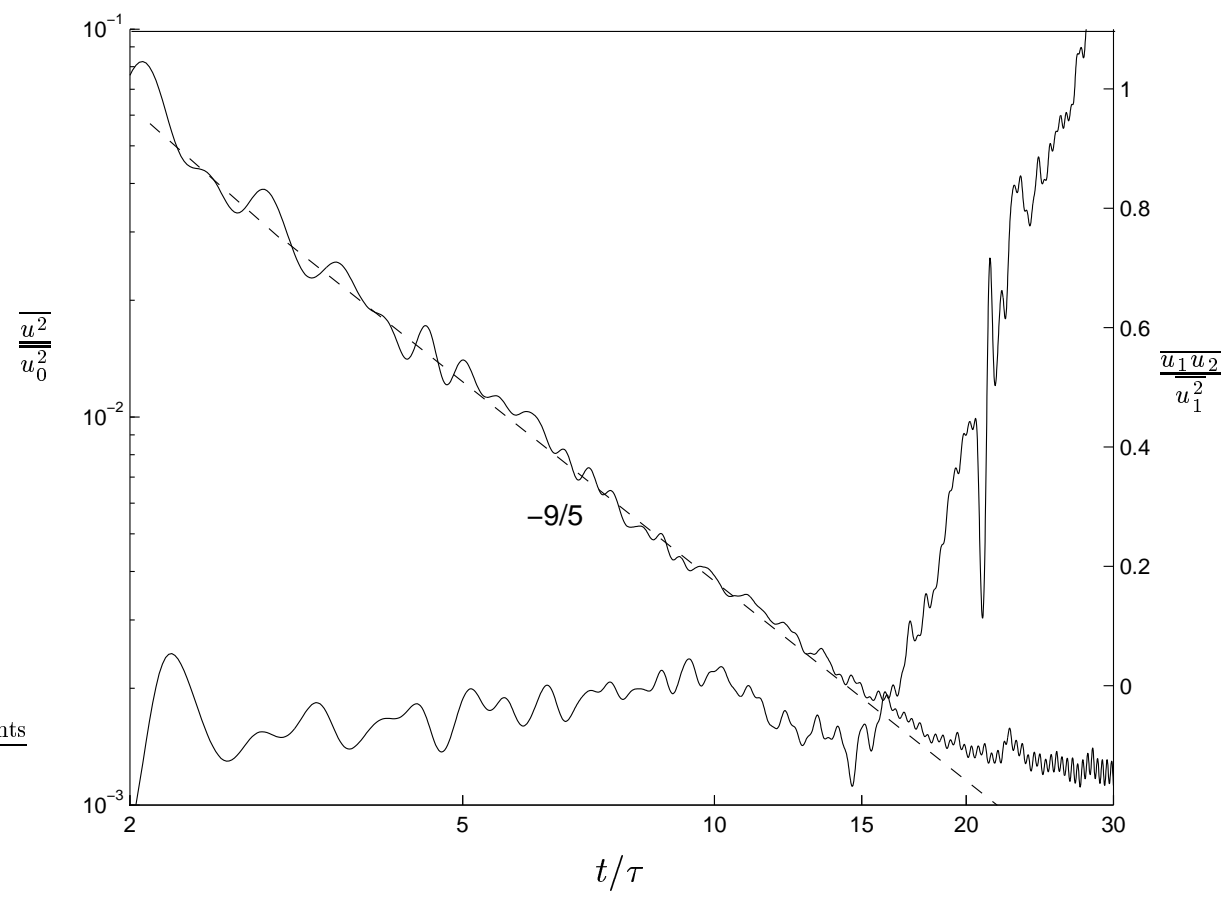

Figure 13: Mean square velocity of a vortex, for $N_{0}=1.0$, and the corresponding velocity correlation coefficients. $B=0.50 T$. The absence of the linear phase may be noted. 


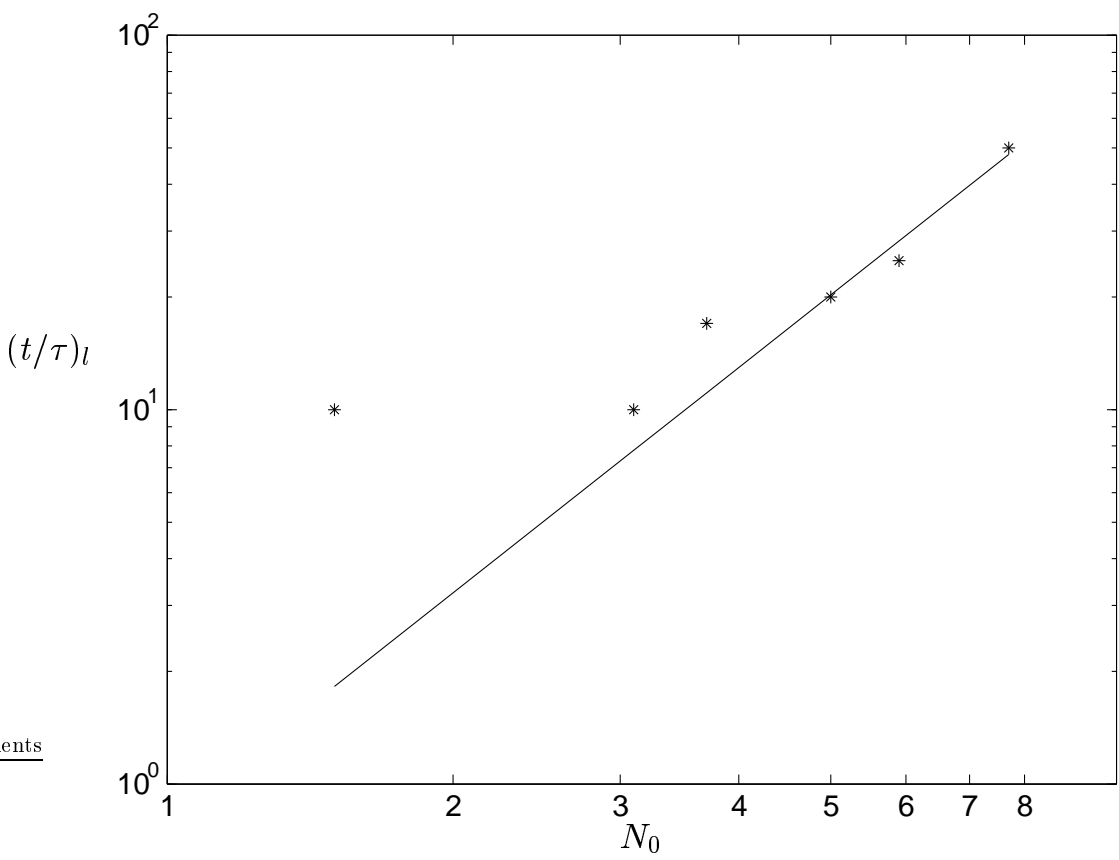

Figure 14: Variation of the linear-non-linear transition time with $N_{0}$, in logarithmic coordinates. The experimental data points are shown by the symbol $*$, and the solid line is the curve $(t / \tau)_{l}=0.8 N_{0}^{2}$. 

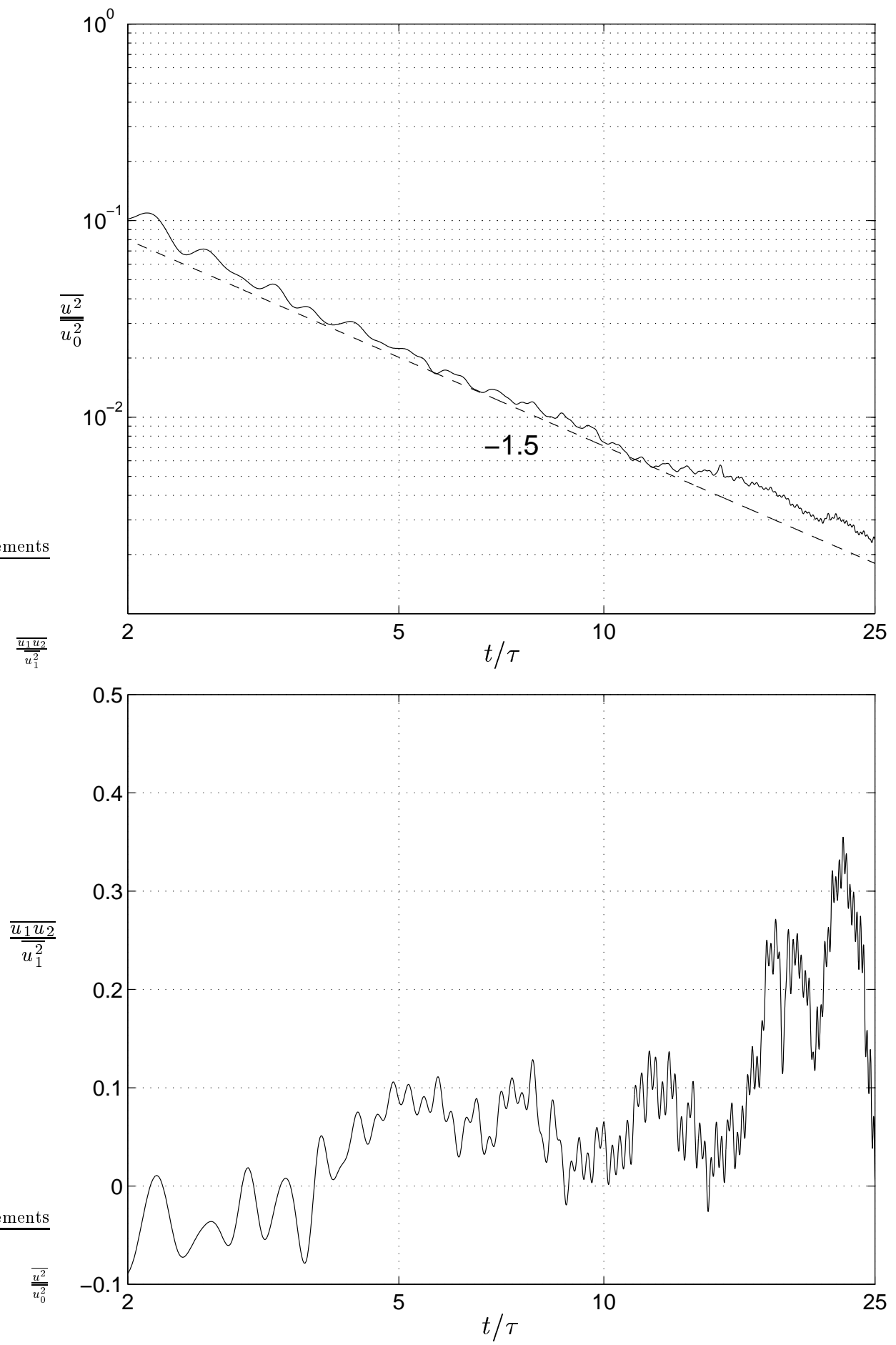

Figure 15: Mean square velocity for $N_{0}=0.7$, and the corresponding correlation coefficients. $B=0.42 \mathrm{~T}$. 